\title{
Review
}

Yueqiang Hu, Xudong Wang, Xuhao Luo, Xiangnian Ou, Ling Li, Yiqin Chen, Ping Yang, Shuai Wang and Huigao Duan*

\section{All-dielectric metasurfaces for polarization manipulation: principles and emerging applications}

https://doi.org/10.1515/nanoph-2020-0220

Received March 31, 2020; accepted May 27, 2020; published online June 29, 2020

\begin{abstract}
Metasurfaces, composed of specifically designed subwavelength units in a two-dimensional plane, offer a new paradigm to design ultracompact optical elements that show great potentials for miniaturizing optical systems. In the past few decades, metasurfaces have drawn broad interests in multidisciplinary communities owing to their capability of manipulating various parameters of the light wave with plentiful functionalities. Among them, pixelated polarization manipulation in the subwavelength scale is a distinguished ability of metasurfaces compared to traditional optical components. However, the inherent ohmic loss of plasmonic-type metasurfaces severely hinders their broad applications due to the low efficiency. Therefore, metasurfaces composed of high-refractive-index all-dielectric antennas have been proposed to achieve high-efficiency devices. Moreover, anisotropic dielectric nanostructures have been shown to support large refractive index contrast between orthogonal polarizations of light and thus provide an ideal platform for polarization manipulation. Herein, we
\end{abstract}

\footnotetext{
*Corresponding author: Huigao Duan, State Key Laboratory of Advanced Design and Manufacturing for Vehicle Body, College of Mechanical and Vehicle Engineering, Hunan University, Changsha 410082, China; and Advanced Manufacturing Laboratory of Micronano Optical Devices, Shenzhen Research Institute, Hunan University, Shenzhen, 518000, China, E-mail: duanhg@hnu.edu.cn.

Yueqiang Hu: State Key Laboratory of Advanced Design and Manufacturing for Vehicle Body, College of Mechanical and Vehicle Engineering, Hunan University, Changsha 410082, China; and Advanced Manufacturing Laboratory of Micro-nano Optical Devices, Shenzhen Research Institute, Hunan University, Shenzhen, 518000, China, E-mail: huyq@hnu.edu.cn

Xudong Wang, Xuhao Luo, Xiangnian Ou, Ling Li and Yiqin Chen: State Key Laboratory of Advanced Design and Manufacturing for Vehicle Body, College of Mechanical and Vehicle Engineering, Hunan University, Changsha 410082, China

Ping Yang and Shuai Wang: Institute of Optics and Electronics, Chinese Academy of Sciences, Chengdu, Sichuan 610209, China
}

present a review of recent progress on all-dielectric metasurfaces for polarization manipulation, including principles and emerging applications. We believe that high efficient all-dielectric metasurfaces with the unprecedented capability of the polarization control can be widely applied in areas of polarization detection and imaging, data encryption, display, optical communication and quantum optics to realize ultracompact and miniaturized optical systems.

Keywords: all-dielectric materials; matasurface; polarization manipulation; polarization detection and imaging; quantum optics; vector vortex beam.

\section{Introduction}

Since the 1960s, Veselago [1] has proposed predictions for lefthanded materials with negative permittivity and permeability. Until the end of the twentieth century, Pendry introduced the idea of metamaterials [2], that is, the materials composed of artificial structural units arranged in a specific form can produce unprecedented electromagnetic properties that do not exist in natural materials. Later, Smith et al. experimentally realized the negative refraction with metamaterials [3]. Since then, the design concept of metamaterials has attracted widespread attention, spawning photonic crystals [4, 5], lefthanded materials $[6,7]$, cloaking $[8,9]$ and other novel optical devices [10-12]. However, it is difficult to achieve many potential applications of metamaterials in optical frequencies due to the challenge of fabricating complex three-dimensional (3D) structures. To avoid this dilemma, metasurfaces, the twodimensional (2D) counterpart of metamaterials, have rapidly emerged as a new paradigm for designing optical elements.

The metasurfaces usually consist of subwavelength structures (meta-atoms) arranged in a 2D plane. By adjusting the shapes, sizes, orientations or positions of meta-atoms, the phase gradient is introduced at the interface for arbitrary molding of the optical wavefront [13-16]. In 2011, Capasso's group [17] first proposed the generalized Snell's law and experimentally demonstrated a two- 
dimensional array of $\mathrm{V}$-shaped plasmonic antennas to form a phase discontinuity at the interface. Anomalous reflection and refraction phenomena were observed with a linear phase gradient along the interface. After that, various optical elements, ranging from beam deflectors [18-20], metalenses [21-22], meta-holograms [23-25], and vortex generators [26-28] have been demonstrated using plasmonic metasurfaces with the resonance phase or geometric phase. However, due to the high intrinsic ohmic loss of plasmonic nanostructures in the optical range, the efficiencies of metasurface-based devices are difficult to improve, which limits their practical applications. Therefore, metasurfaces composed of high-refractive-index and all-dielectric antennas, in which light does not couple to plasmons have been introduced to achieve nanophotonic devices with high efficiencies [29-33]. It bridges the gap between fundamental nanophotonic science and practical device by overcoming the critical issue of optical absorption. In addition, the fabrication of metasurface is compatible with the complementary metal oxide semiconductor (CMOS) fabrication process and thus holds great promises to realize more compact optical systems with broad application prospects [34-36]. In Figure 1a, we summarize the complex refractive indices of some typical dielectric materials for constructing all-dielectric metasurfaces (ADMs). These materials have high values of the real part of the complex refractive index, providing a great refractive-index contrast between the dielectric materials and air for large accumulated phase shifts in the wavelength-scale thickness and also large refractive index contrast between orthogonal axes for polarization manipulation. Considering the extinction coefficient, the applicable wavelengths of different materials should be carefully chosen for high transmission efficiencies. For example, $\mathrm{Si}$ is an excellent dielectric material in the infrared and even terahertz bands. In the visible light band, metal oxides and nitrides such as $\mathrm{TiO}_{2}, \mathrm{Ta}_{2} \mathrm{O}_{5}, \mathrm{HfO}_{2}$ and
$\mathrm{Si}_{3} \mathrm{~N}_{4}$ are preferable candidates. In the ultraviolet band, the absorption of materials is large so that the dielectric material should be chosen by balancing the loss.

Besides the phase manipulation, taking advantages of strong light-matter interactions at the subwavelength scale, metasurfaces also show a powerful capability to regulate light with different degrees of freedom (DoFs) such as amplitude [37-42], frequency [43, 44], chromatic dispersion [45] and polarization [46], which have drawn much attention of the scientific community in the last few years. For example, utilizing the electric dipole resonance, magnetic dipole resonance or guided mode resonance in the artificial antennas to manipulate the amplitude, perfect absorbers [47, 48], structural colors [49, 50], amplitudebased holography [51] have been demonstrated. In addition, metasurfaces are also used for nonlinear optics applications for frequency manipulation, such as nonlinear generations [52] (e.g., second harmonic generation and high harmonic generation), optical frequency mixer [53], beam shaping [54], nonlinear metalenses [55], nonlinear meta-holograms [56]. Chromatic dispersion engineering with metasurfaces is applied to multiwavelength [57], narrowband, and broadband achromatic [58, 59], spectroscopy [60] and color holograms [61].

Among them, polarization manipulation at the subwavelength is the unique capability of ADMs by engineering the anisotropy of the media compared to traditional refractive and diffractive optical components [62]. The principal refractive indices along two orthogonal axes (extraordinary and ordinary) rarely exceed $10 \%$ in natural materials, while ADMs can be engineered to have much higher refractive index contrast by introducing asymmetric meta-atoms [63]. Acting as birefringent elements, the meta-atoms with specific design can be utilized to realize subwavelength pixelated polarization control for polarization conversion, polarization dependent multiplexing and even complex vector beams [64]. On this basis,

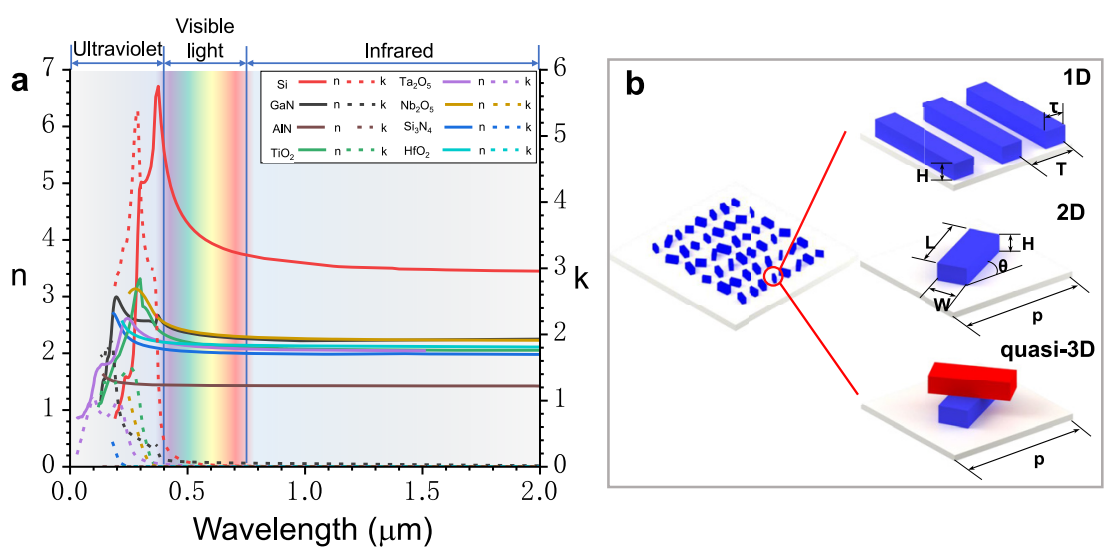

Figure 1: Materials and general design of alldielectric metasurfaces. (a) The complex refractive index of the typical dielectric materials for constructing all-dielectric metasurfaces in different optical wavelength bands. (b) Schematics of alldielectric metasurfaces for polarization manipulation. 
combined with phase and amplitude regulation, more complex functions can be realized [65]. Because polarization contains abundant valuable information (e. g. material properties, surface topology information, biological tissue properties, optical activity, and quantum information), metasurfaces with powerful polarization manipulation ability can be applied to many areas such as remote sensing, biology, medicine, microscopy, optical communication and quantum science to achieve an ultracompact and miniaturized optical system. In the past years, numerous efforts have been made to fully utilize this ability, and various new functions and emerging applications have been demonstrated.

In this paper, we aim to review the noticeable research achievements over the last few years focusing on polarization manipulation by ADMs. Following the introduction in Section 1, the rest of this review contains the following three sections. In Section 2, the polarization expression, the principles of basic polarization manipulation and polarization-related functionalities realized by "polarization plus" are summarized. Various applications based on these principles, such as polarization conversion, beam splitters, metalens, nano-printing, holography, polarization imaging and detection, vector vortex beam and quantum optics, will be reviewed in Section 3. In the final section, a summary and outlook are provided for further developments of the ADMs related to the polarization manipulation.

\section{Principles}

\subsection{The description of polarization}

In classic electromagnetics, light is a kind of transverse wave, the vibration direction of the light field vector is perpendicular to the propagation direction of the light wave. Generally, in the vibration plane, the vibration direction of the light field is asymmetric relative to the propagation direction of the light wave. This property is called the polarization property of light waves. It is also a remarkable sign that the transverse wave different from the longitudinal wave. According to the projection shape of the vibration plane on the wavefront, the polarization can be divided into linear polarization, circular polarization and elliptic polarization. A scalar light field has a consistent distribution of polarization. In contrast, the light field with uneven polarization distribution is a vector light field. While in quantum physics, light is composed of photons, which are bosons that carry energy, momentum, and mass. Photons are spin-1 particles and the two spin states of \pm 1 correspond to the right circular polarization (RCP) and left circular polarization (LCP). They carry spin angular momentum (SAM) per photon and the general state of polarization (elliptical) is a superposition of them.

The polarization of light in classic optics is often expressed mathematically by vector form (Jones vector and Stokes vector). In 1941, Jones [66] used a vector to represent the $x$ and $y$ components of the electric vector,

$$
E=\left[\begin{array}{c}
E_{x} \\
E_{y}
\end{array}\right]=\left[\begin{array}{c}
E_{0 x} \mathrm{e}^{\mathrm{i} \varphi_{x}} \\
E_{0 y} \mathrm{e}^{\mathrm{i} \varphi_{y}}
\end{array}\right]
$$

where $E_{0 x}, E_{0 y}$ and $\varphi_{x}, \varphi_{y}$ are the amplitudes and corresponding phases along two axes, respectively. Jones vector represents the amplitudes and phases of two orthogonal components of light at the same time and the same position, regardless of time, and can only be used for fully polarized light.

In 1852, Stokes proposed to use four parameters to express the intensity and state of polarization (SOP) of a light wave, as shown below,

$$
S=\left[\begin{array}{c}
S_{0} \\
S_{1} \\
S_{2} \\
S_{3}
\end{array}\right]=\left[\begin{array}{c}
E_{x}^{2}+E_{y}^{2} \\
E_{x}^{2}-E_{y}^{2} \\
2 E_{x} E_{y} \cos \delta \\
2 E_{x} E_{y} \sin \delta
\end{array}\right]=\left[\begin{array}{c}
I_{x}+I_{y} \\
I_{x}-I_{y} \\
I_{45^{\circ}}-I_{-45^{\circ}} \\
I_{R}-I_{L}
\end{array}\right]
$$

where, $\delta$ represents the phase difference between the $x$ and $y$ components. In this formula, Stokes parameters can be expressed in relation to several typical field intensities. Specifically, $S_{0}$ is the total light intensity, $S_{1}$ is the light intensity difference of the two components after splitting the light along the $x$ and $y$ directions, $S_{2}$ is the light intensity difference of the two components after splitting the light along the $45^{\circ}$ and $135^{\circ}$ directions, and $S_{3}$ is the light intensity difference between the two components after splitting the light into RCP light and LCP light. According to this formula, by measuring the intensity of these polarization states, we can obtain Stokes parameters and calculate the polarization of the light. The advantage of this method is that it can represent not only fully polarized light but also partially polarized and natural light.

In the expression of quantum mechanics, a photon has two spin states $\sigma_{ \pm}$which carry the SAM $L_{z}$, depending on the RCP and LCP state of polarization. And a paraxial, circularly polarized helical beam carries a total angular momentum (TAM) per photon, which can be expressed by

$$
L_{z}=\hbar \sigma_{ \pm}=\left\{\begin{array}{l}
+1 \hbar, \mathrm{RCP} \\
-1 \hbar, \mathrm{LCP}
\end{array}, \quad \text { where } \sigma_{ \pm}=\left\{\begin{array}{l}
+1, \mathrm{RCP} \\
-1, \mathrm{LCP}
\end{array},\right.\right.
$$


where $\hbar$ is Planck's constant $h$ divided by $2 \pi$. All other polarization states are superpositions of these two states, which can be expressed by

$$
|\Psi\rangle=A_{1}^{*}\left|\sigma_{+}\right\rangle+A_{2}^{*}\left|\sigma_{-}\right\rangle
$$

where $A_{1}^{*}, A_{2}^{*}$ are the complex coefficients that provide both a weight and a phase to the two TAM states. Note that the orbital angular momentum (OAM) is not considered here.

\subsection{Mechanisms of polarization manipulation with all-dielectric metasurfaces}

As mentioned above, polarized light can be decomposed into linear components along two orthogonal directions. To achieve polarization control, it is necessary to manipulate the phase and amplitude of the light in these two directions. The general schematic illustration of birefringent dielectric metasurfaces is shown in Figure 1b. They are usually composed of an array of subwavelength anisotropic metaatoms with fixed height, which can be 1D gratings with a single parameter, 2D nanoposts with different lengths, widths and orientations or even quasi-3D structures with few layers of nanoposts. The meta-atoms are placed at the centers of periodic unit cells. Subwavelength anisotropic structures can support large refractive index contrast for transverse electric (TE) and transverse magnetic (TM) polarized light. As a result, each meta-atom can generate two independent phase shifts of transmitted light in two directions. Due to the high refractive index, light is mainly confined inside the meta-atoms so that the light scattered by each meta-atom is primarily determined by the geometrical parameters and has negligible dependence on its neighboring meta-atoms. This illustrates the feasibility of birefringent metasurfaces. The birefringent dielectric metaatom can be represented by a Jones matrix

$$
\mathbf{J}=\left[\begin{array}{cc}
J_{x x} & J_{x y} \\
J_{y x} & J_{y y}
\end{array}\right]
$$

where $J_{x x}, J_{x y}, J_{y x}, J_{y y}$ are elements of the Jones matrix complex numbers. The relationship between the input and output light fields can be expressed as $\mathbf{E}^{\text {out }}=\mathbf{J} \mathbf{E}^{\text {in }}$, where, $\mathbf{E}^{\text {in }}$ is an incident optical wave with spatially varying electric field, and $\mathbf{E}^{\text {out }}$ is the output electric field that we need. Take the rectangular meta-atom with different dimensions and orientations in Figure $1 \mathrm{~b}$ as a metasurface platform, the matrix is decomposed into eigenvalues and eigenvectors as follows,

$$
\mathbf{J}=\mathbf{R}(\theta)\left[\begin{array}{cc}
t_{x} & 0 \\
0 & t_{y}
\end{array}\right] \mathbf{R}(-\theta)
$$

where $t_{x}$ and $t_{y}$ are, respectively, the complex scattering coefficients for incident light linearly polarized along the two axes of the meta-atom which determined by the dimensions of the meta-atom along the two axes. $\mathbf{R}(\theta)$ is the rotation matrix with an orientation of $\theta$ which can be expressed by $R(\theta)=\left[\begin{array}{ll}\cos \theta & \sin \theta \\ -\sin \theta & \cos \theta\end{array}\right]$. Therefore, any symmetric and unitary Jones matrix can be obtained utilizing a metasurface with at least three parameters if its $t_{x}$ and $t_{y}$ and in-plane orientation angle $\theta$ can be designed freely.

\subsection{Realization of functionalities with "polarization plus"}

As introduced above, ADMs have the distinguished ability to modulate the polarization in the subwavelength pixels. Based on this, basic polarization conversion and generation of complex vector light field can be realized. However, to achieve more abundant polarization-related functionalities, other DoFs of parameter manipulation should be fully combined such as phase and amplitude manipulation to achieve customized shaping of the near-field or far-field wavefront. Since polarization contains amplitude and phase information, it is natural to couple them with the polarization control. Figure 2 shows a framework diagram of what we call "polarization plus". We have divided the present studies into polarization-only manipulation, polarization and phase comanipulation, polarization and amplitude co-manipulation, and polarization, phase and amplitude co-manipulation. Numerous functions and applications have been developed based on this framework. Meanwhile, we believe that more principles and applications of "polarization plus" will be discovered in the future. In the following, the typical principles of realizing "polarization plus" will be summarized.

The geometric phase (or Pancharatnam-Berry phase) is a powerful platform for polarization and phase comanipulation. It means that an additional phase related to the orientation angle $\theta$ of the meta-atom is generated when the circularly polarized light beam $\mathbf{E}^{\mathrm{in}}=\left[\begin{array}{c}1 \\ \pm i\end{array}\right]$ passes through the metasurface. According to Eq. (6), the output light can be derived by multiplying the Jones matrix as

$$
\mathbf{E}^{\text {out }}=\mathbf{J E}^{\text {in }}=\frac{t_{x}+t_{y}}{2}\left[\begin{array}{c}
1 \\
\pm i
\end{array}\right]+\frac{t_{x}-t_{y}}{2} \exp (\mp i 2 \theta)\left[\begin{array}{c}
1 \\
\mp i
\end{array}\right]
$$

The first term on the right side of Eq. (7) represents the circularly polarized light wave with the same chirality as the incident light and the second term represents the circularly polarized light wave with opposite chirality and 


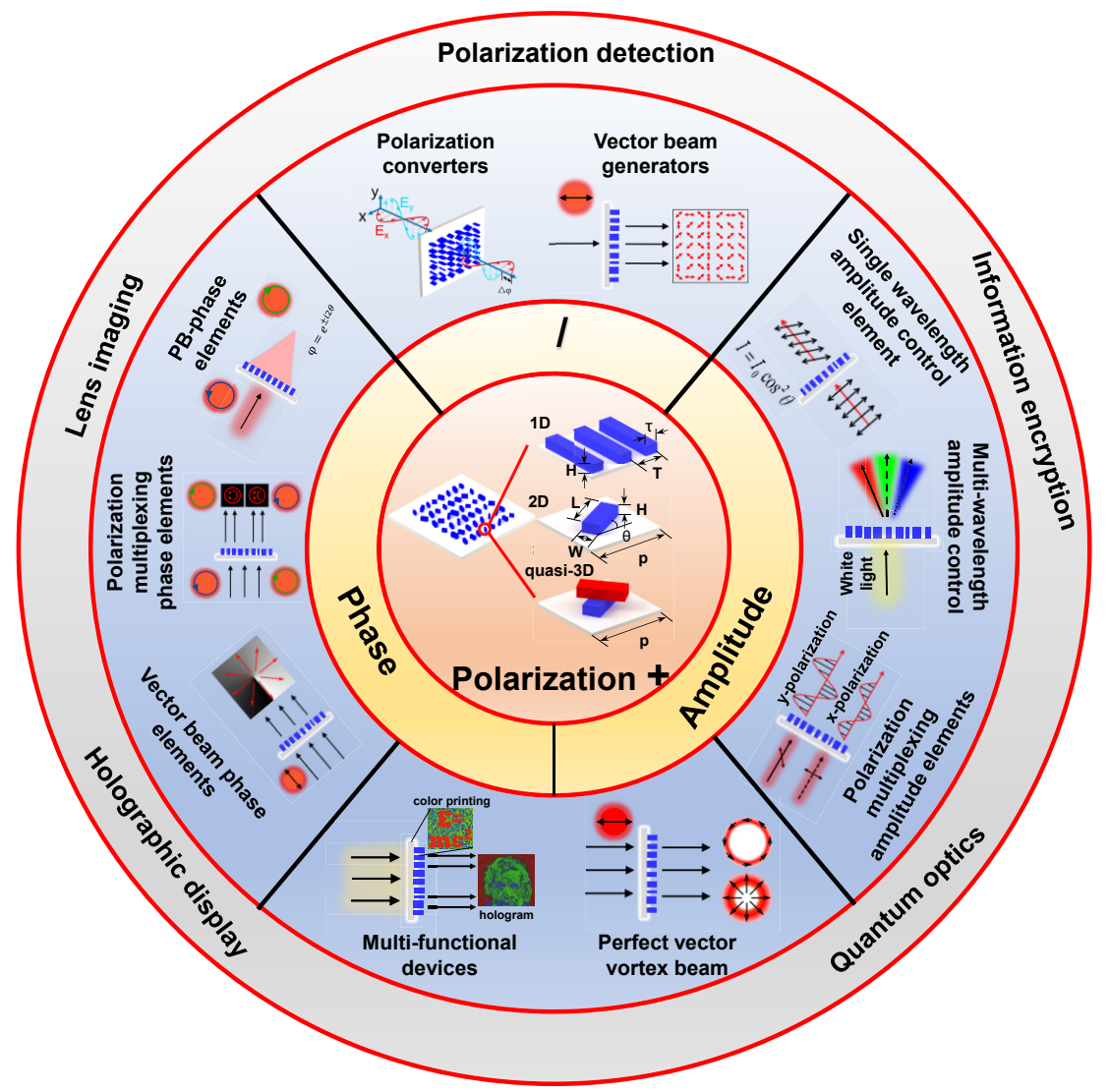

Figure 2: Framework diagram of "polarization plus" for achieving polarization-based functions and applications.

an additional geometric phase of $2 \theta$. If the meta-atom acts as a half-waveplate, only the second term can be maintained, and its phase can cover $0-2 \pi$ range if the post is rotated from $0^{\circ}$ to $180^{\circ}$. This allows us to achieve specific wavefront shaping through the additional phase while implementing polarization conversion to achieve specific functions, such as beam splitters, lenses, and phase holograms. In addition, the additional geometric phase is chiral-conjugated, which also makes it possible to implement chiral-dependent multiplexing phase devices. On the other hand, in quantum mechanics, the RCP and LCP state of polarization correspond to the two spin states of phonon which carry the SAM as described above. Besides, light waves with an azimuth-dependent phase distribution $l \phi$, where $\phi$ is the azimuthal angle and $l$ is any integer, carry OAM. Therefore, geometric phase elements provide a natural connection between SAM and OAM which shows great potentials of applications in the quantum optics. They perform the transformation $\left|\sigma_{-}\right\rangle \rightarrow e^{2 l \phi}\left|\sigma_{+}\right\rangle$and $\left|\sigma_{+}\right\rangle \rightarrow e^{-2 l \phi}\left|\sigma_{-}\right\rangle$when the orientations are chosen to be $\theta=l \phi / 2$. It means that the LCP and RCP are converted to output states with opposite spin and $\pm 2 l \hbar \mathrm{OAM}$, which is called spin-orbit-conversion (SOC).

In addition to the geometric phase, propagation phase or resonant phase can also be used to achieve comanipulation of polarization and phase. If meta-atoms are arranged with no rotation, the Jones matrix can be simplified by

$$
\mathbf{J}=\left[\begin{array}{ll}
e^{i \phi_{x}} & 0 \\
0 & e^{i \phi_{y}}
\end{array}\right]
$$

where $\phi_{x}$ and $\phi_{y}$ are the propagation phase or resonant phase shifts on linearly polarized light along its two axes. It means that the output electric field can be obtained by $\left[\begin{array}{c}e^{i \phi_{x}} \\ 0\end{array}\right]$ and $\left[\begin{array}{c}0 \\ e^{i \phi_{y}}\end{array}\right]$ under $x$ - and $y$-polarized light, respectively. Therefore, a single non-interleaved metasurface can impose two distinct phase profiles under two orthogonal incident polarizations to achieve polarization-dependent multiplexing phase elements.

However, there is limited space for polarization manipulation using the geometric phase with angular DoF only or the propagation phase with structural dimensional 
DoF only. Therefore, complete manipulation of polarization and phase can be achieved by making full use of the flexibility of the Jones matrix which can also be seen as both use of geometric phase and propagation phase. For a symmetric and unitary Jones matrix, we have $J_{x y}=J_{y x}$ and $J_{x x} J_{x y}^{*}+J_{y x}^{*} J_{y y}=0$, and then we can get [67]

$$
\left[\begin{array}{cc}
E_{x}^{\text {out } *} & E_{y}^{\text {out } *} \\
E_{x}^{\text {in }} & E_{y}^{\text {in }}
\end{array}\right]\left[\begin{array}{c}
J_{x x} \\
J_{y x}
\end{array}\right]=\left[\begin{array}{c}
E_{x}^{\text {in } *} \\
E_{x}^{\text {out }}
\end{array}\right]
$$

where * represents complex conjugation. Therefore, $J_{x x}$ and $J_{y x}$ can find from this equation for given input and output polarizations. And $J_{y y}$ can be obtained from $J_{y y}=-\exp \left(2 i<J_{y x}\right) J_{x x}^{*}$. It means that a unitary and symmetric Jones matrix can be always found that transforms any input light field $\mathbf{E}^{\text {in }}$ to any output light field $\mathbf{E}^{\text {out }}$. This provides great freedom in achieving arbitrary polarization distribution while also achieving arbitrary phase distribution for applications in vector vortex beam generation, more complex multiplexing elements and even arbitrary SOC in quantum optics.

For polarization and amplitude co-manipulation, there are generally two strategies. First, when a linearly polarized light beam passes through a polarizer, the intensity of the transmitted light can be tailored by the angle $\alpha$ between the axis of the polarizer and the polarization direction of the incident light. This is called Malus's law, which can be expressed by

$$
I=I_{0} \cos ^{2} \alpha
$$

where $I_{0}$ is the intensity of incident light. As described above, the all-dielectric meta-atom can regulate the amplitude and phase of the long and short axes to construct as a waveplate or a polarizer. Therefore, the vector light field can be generated by the meta-atoms to achieve the amplitude control by combining the polarizer at the output end. Furthermore, by directly constructing the meta-atoms as polarizers, it is possible to obtain the amplitude manipulation of the vector field without the polarizer.

Second, the amplitude can be controlled by the crosspolarization conversion efficiency (CPCE) from the incident polarization to the cross polarization in the geometric phase manipulation. From Eq. (7), the coefficient of the second term on the right side of the equation $\frac{t_{x}-t_{y}}{2}$ is a complex amplitude, whose amplitude reflects the CPCE and phase is a propagation phase superimposed on the geometric phase. By controlling the phase of the long and short axes and implementing them with a circular polarization analyzer at the output end, the amplitude can be adjusted continuously. However, the strategies introduced above are all for single-wavelength polarization and amplitude co-manipulation. Many previous studies have shown that the CPCE of the geometric phase has a certain selectivity in the wavelength dimension. Using this property, in combination with the above two single-wavelength amplitude modulation schemes, amplitude modulation at multiple wavelengths can also be achieved. With these polarization-based amplitude modulations, applications of encryption for hiding a grayscale image in the polarization distribution, as well as amplitude-holograms can be realized.

For polarization plus phase and amplitude comanipulation, if the phase is taken into account in the above polarization and amplitude co-manipulation schemes, and the amplitude and phase information are decoupled by designed algorithms, the co-manipulation of polarization, amplitude, and phase can be achieved for complex functions such as holography and nano-printing multiplexing. However, the freedom of regulation of polarization in this scheme is greatly limited. Therefore, in the latest research, some researchers have explored the use of multi-unit scheme to provide more parametric DoFs and inverse design to achieve arbitrary independent regulation of polarization, phase, and amplitude for applications such as perfect vortex vector beams and polarization-dependent complex holography.

\section{Applications}

Utilizing the manipulation schemes under "polarization plus" framework of ADMs introduced above, a variety of applications have been spawned ranging from lens imaging, holographic display, data storage and encryption, polarization detection to quantum optics and other multifunctional elements to achieve miniaturized and versatile optical systems. In what follows, we review some exciting progress of relative applications.

\subsection{Polarization converters}

The basic applications using the fundamental polarization manipulation ability are the polarization generation and conversion corresponding to the elements of polarizers and waveplates. Conventional polarization control using crystals that allow specific polarized light to pass through or have different refractive indices in orthogonal directions (birefringence) are bulky and lack of flexibility. Similar to the birefringent crystal, the subwavelength dielectric anisotropic structures can generate different phase shifts 
along two orthogonal axes, while the phase shifts between the two directions can be several orders of magnitude higher in the same thickness due to the large index contrast between dielectric material and air. The phase difference between the two electric field components can be freely adjusted by the geometry parameters, leading to an ultrathin waveplate with an arbitrary phase delay.

Various all-dielectric polarization conversion metasurfaces for quarter-waveplates, half-waveplates or even waveplates with arbitrary delay have been proposed with different phase regulation mechanisms [68-77]. Lin et al. proposed and experimentally demonstrated an ultrathin waveplate with Si-based gradient metasurface [78]. A 120-nm-wide and 100-nm-high 1D nanobeam was designed to support a strong resonance under TE illumination (with the electric field polarized normal to the length of the structure) and the orthogonal TM polarization in the 550-nm wavelength range as Figure 3a shown. Its resonant behavior results in a $0.14 \pi$ phase shift for TE-polarized light and a $1.15 \pi$ phase shift for TM-polarized light leading to a halfwaveplate. However, the phase difference of the waveplate varies from $0.4 \pi$ to $1.2 \pi$ with the wavelength from 490 to $700 \mathrm{~nm}$ resulting in a narrowband device. Still using the one-dimensional structure, Zhang et al. [79] designed a broadband (600-1000 nm) quarter-waveplate and a halfwaveplate using the $\alpha \mathrm{TiO}_{2}$ grating array (Figure 3b). This is because the real part of the refractive index of $\alpha \mathrm{TiO}_{2}$ remains consistent from $\lambda=550 \mathrm{~nm}$ to $\lambda=1000 \mathrm{~nm}$. It means that there almost no material dispersion in this range which allows one to design a waveplate with a broadband response in the visible range. Utilizing similar principles, Yang et al. experimentally demonstrated a broadband reflection-mode polarization converter in the near-infrared band with 2D structured Si rectangular resonators on a silver ground plane as Figure 3c shown [68]. Mie resonances are utilized in the $500 \mathrm{~nm}$-long, $250 \mathrm{~nm}$-wide and $380 \mathrm{~nm}$-high Si metaatoms and the multiple reflections in the spacer layer of the metasurface relax the requirement of aspect ratio and the height of the resonators. It realizes a function of halfwaveplate across a $200 \mathrm{~nm}$ bandwidth with over $98 \%$ CPCE and near-unity reflectance. In addition to the half waveplate, Kruk et al. also demonstrated a metasurface for the quarter-waveplate in a broadband range [72] (Figure 3d). The metasurface is composed of spaced silicon nanopillars with a dimension of $310 \mathrm{~nm}$ (length), $370 \mathrm{~nm}$ (width), $850 \mathrm{~nm}$ (height) in a $750 \mathrm{~nm}^{2}$ period allowing more multipolar modes for the quarter-waveplate. By overlapping the scattering contributions of both electric and magnetic of $\mathrm{Si}$ elliptical meta-atoms, the ultrahigh transmission across a wide bandwidth is realized. Experimental results show 90\% transmission and $~ 99 \%$ CPCE across several telecommunication bands. Such a high conversion efficiency is at least three times greater than conventional birefringent media. ADMs can be designed not only for polarization generation or conversion, but also as a depolarizer. Wang et al. [80] proposed a spatial domain depolarizer based on $\mathrm{TiO}_{2}$ metasurface which can efficiently depolarize linearly polarized light with a degree of polarization of less than $10 \%$ from 450 to $670 \mathrm{~nm}$ (Figure 3e). The meta-atoms in the depolarizer act as half waveplates to rotate the incident linear polarization. And the fast axes of these nanoscale waveplates in the metasurface are chosen to be random so that an incident linearly polarized light can be transformed into random polarization directions to significantly reduce its degree of polarization. Due to high pixel density benefited from the subwavelength scale of the meta-atoms, it has been proven that the metasurface-based depolarizer can depolarize the light beam with a diameter down to $10 \mu \mathrm{m}$ which is about two orders of magnitude smaller than commercial liquid-crystal-based depolarizers.

\subsection{Polarization beam splitters}

The polarization beam splitter (PBS) can split an incident beam into two orthogonally polarized beams, which can be applied in optical fiber telecommunications and polarization imaging. Traditional PBS based on birefringent crystals is bulky and will hinder its wide application in integrated systems. With the polarization and phase comanipulation, ADM can achieve polarization-sensitive phase gradients, which is an ideal platform for compact PBS. On the one hand, the chiral-conjugation of geometric phase can be utilized to naturally split the beam to two orthogonally circular polarized light. Khorasaninejad et al. [81] first constructed a metasurface-based chiralitydistinguishing beam splitter based on amorphous silicon (a-Si) nanofins, as shown in Figure 4a. The metasurface is composed of unit cells which are formed by six nanofins acting as the birefringent elements which successively rotate by $30^{\circ}$ with a pitch of $0.5 \mu \mathrm{m}$. By deriving, they found that the power of the $m=0$ diffracted order does not depend on the incident chirality while the powers of the $m= \pm 1$ orders are sensitive to the chirality, enabling to identify the handedness of the incident light. This can be understood from the geometric phase which can generate the chirality-conjugated phase $\phi= \pm 2 \theta$ (the sign determined by the incidence/transmission polarizations, with the + sign for LCP/ RCP and the - sign for RCP/LCP) related to the orientation $\theta$ of the nanofin [82]. Utilizing this principle, Zhou et al. [83] proposed a similar geometric phase metasurface-based PBS for optical edge 


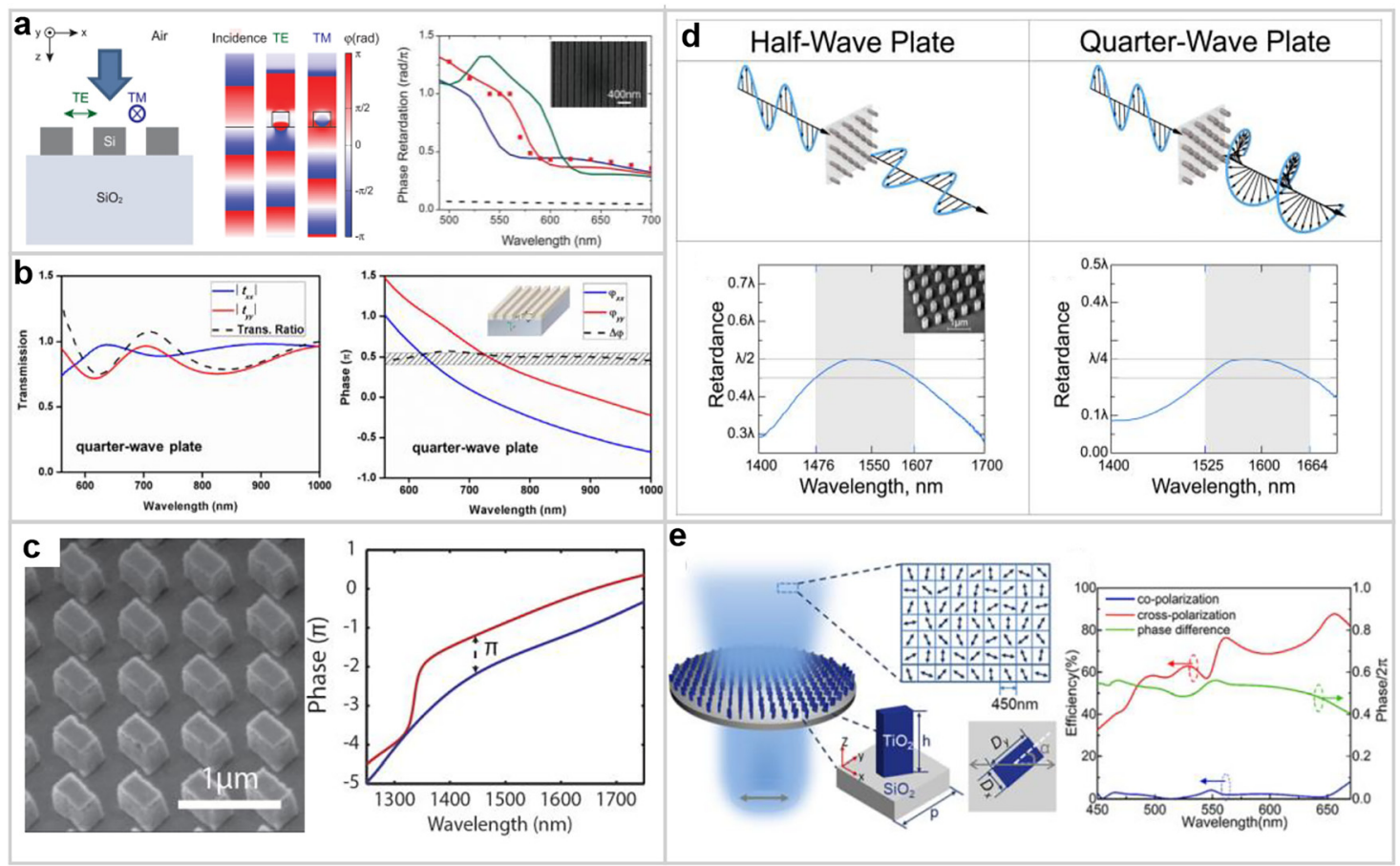

Figure 3: Applications of polarization convectors with all-dielectric metasurfaces. (a) A Si nanobeam was chosen to support a strong resonance under transverse electric (TE) illumination and the orthogonal transverse magnetic (TM) polarization [78]. (b) A broadband (600$1000 \mathrm{~nm}$ ) quarter-waveplate using the dispersionless $\alpha_{\mathrm{TiO}_{2}}$ grating array [79]. (c) A broadband reflection-mode dielectric metasurface for the half-waveplate with structured Si rectangular resonators on a silver ground plane [68]. (d) All-dielectric metasurfaces for half-waveplate and quarter-waveplate in a broadband range [72]. (e) A spatial domain depolarizer based on $\mathrm{TiO}_{2}$ metasurface in the visible range [80]. Reprint permission obtained from $[68,72,78-80]$.

detection as shown in Figure 4b. The chiral-conjugated geometric phase leads the opposite phase gradient and splitting the same distance with opposite directions for the LCP and RCP components. Therefore, these two light beams are superimposed into the original polarization in the overlapping area but maintain the polarization states at the edges. Through the filtering of the analyzer with axis perpendicular to the incident light, edge detection is realized. However, the above devices can only work in transmission mode. Li et al. [84] designed a silicon geometric phase metasurface-based PBS that can work in both transmission and reflection modes to achieve fullspace beam splitting as Figure 4c. By carefully designing the birefringent unit, a comparable transmittance and reflectance are achieved at operational wavelength of $630 \mathrm{~nm}$. Meanwhile, the CPCE of the two modes can reach $27 \%$ and the original polarized light is suppressed to below 3\%. By utilizing two interleaved geometric phase units perpendicular to each other, the functionality of the blazed grating to split the incident beam into $2 \times 2$ spot arrays with uniform intensity in both transmission and reflection spaces is achieved.
On the other hand, the beam splitting of linearly polarized light based on the ADM has also attracted extensive attention. Khorasaninejad et al. [85] first explored a polarization splitting method for linearly polarized light at pixel level with amorphous-Si nanoridges-based metasurface (Figure 4d). Such splitter can deflect light polarized parallel to nanoridge length into off-axis directions while proceeds light polarized perpendicular to the nanoridge length to the center. This can be understood by considering the nanoridge as a slab waveguide in which interference between light depends on the phase difference between the light paths coupled to its guided mode and propagates adjacent to the nanoridge. For a light wave polarized parallel to nanoridge length, the phase difference can be designed as an odd multiple of $\pi$, which causes a strong destructive interference and deflection of light. Furthermore, the propagation phase or resonant phase along the fast and short axis of the meta-atom can also be used to generate polarization-dependent phase profiles that can split the incident light to two orthogonal linearly polarized light. As shown in Figure 4e, Guo et al. [86, 87] designed a propagation phase-based PBS at the wavelength of $1500 \mathrm{~nm}$ 

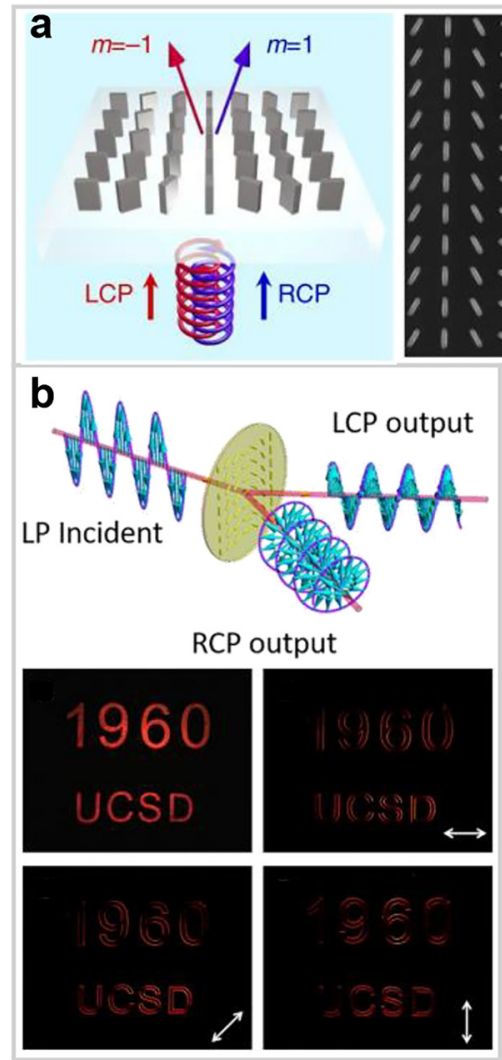
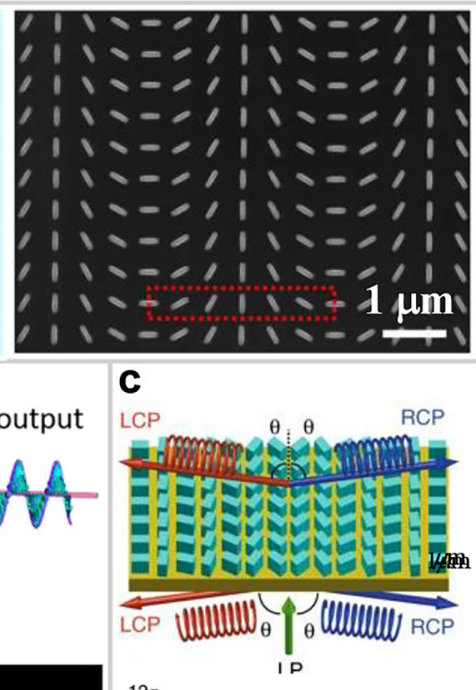
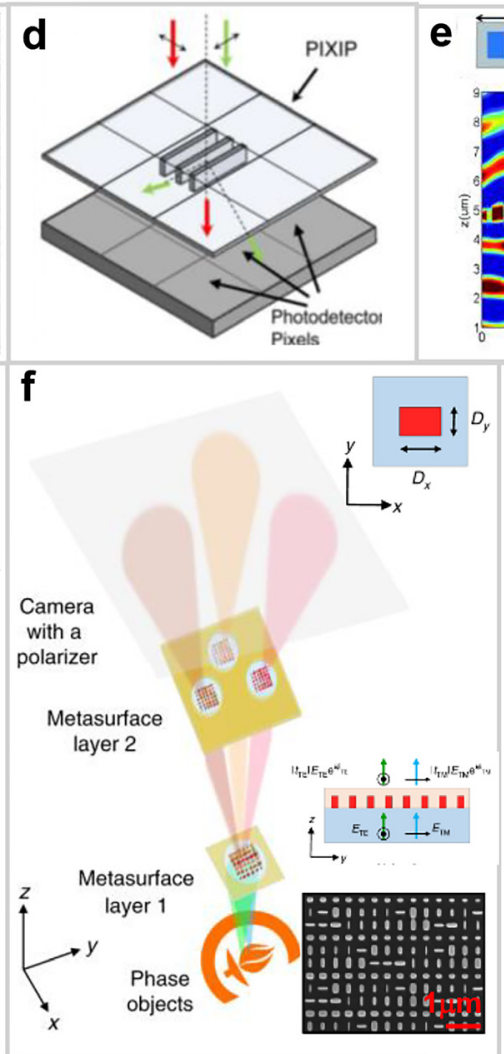

e

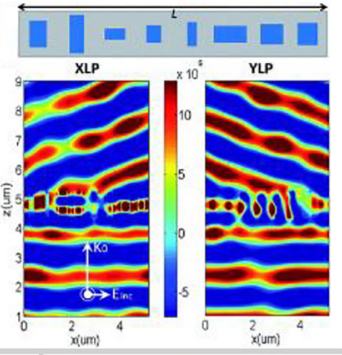

g

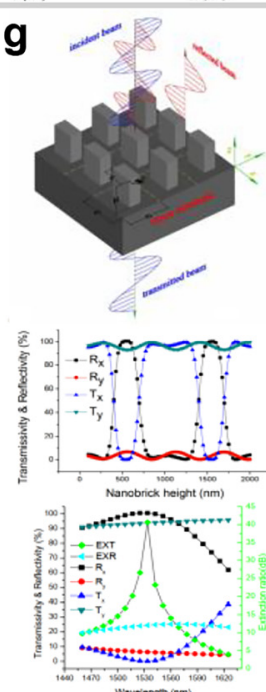

Figure 4: Applications of polarization beam splitters (PBSs) with all-dielectric metasurfaces. (a) A metasurface-based chirality-distinguishing beam splitter based on the chirality-dependent geometric phase of amorphous silicon (a-Si) nanofin [81]. (b) A geometric phase metasurfacebased PBS for optical edge detection [83]. (c) A Si geometric phase metasurface-based PBS in both transmission and reflection modes for fullspace beam splitting [84]. (d) A linearly polarized light splitter at pixel level with amorphous-Si nanoridges-based metasurface to deflect light polarized parallel to nanoridge length into off-axis directions while proceeds light polarized perpendicular to the nanoridge length to the center [85]. (e) Propagation phase-based PBSs supplying two distinct phase gradients for the $x$-linear-polarization and $y$-linear-polarization [86]. (f) A polarization-sensitive dielectric metasurface system for quantitative phase gradient measurements [89]. (g) A Si metasurface-to split two orthogonal linear polarizations into reflection and transmission spaces [90]. Reprint permission obtained from [81, 83-86, 89, 90].

with a Si metasurface. The asymmetric nanobricks can supply two distinct transmission phases for the $x$-linearpolarization and $y$-linear-polarization incidence respectively. Therefore, it can generate two different phase gradients for splitting two orthogonal input polarizations with over $90 \%$ transmitted efficiency. Similarly, Li et al. [88] proposed a Si metasurface-based PBS by directly superimposing two nanoposts with distinct linear polarization phase response to form a cross-shaped meta-atom. Such meta-atom arrays can also support two opposite transmission phase gradients for separating the $x$ - and $y$-polarized light. Using the linear polarization beam splitting can achieve some more complex functions. Kwon et al. [89] propose a system of multifunctional metasurfaces as a microscope for single-shot measurements of quantitative phase gradient, as shown in Figure 4f. The system is composed of two cascaded metasurface layers. By applying linear polarization as above and also spatial multiplexing schemes, layer 1 captures two images for $x$ - and $y$-linear polarizations with focus separated along the $y$-axis and it then splits the incident light equally into three separate directions into the three metalenses in layer 2. By this way, three DIC images are formed with three different phase offsets between the $x$ - and $y$-linear polarizations. This clearly demonstrates the potential of dielectric metasurfaces in miniaturized quantitative phase imaging systems. Unlike the studies of linear beam splitters that are only in transmission space, Zheng et al. [90] explored splitting two orthogonal linear polarizations into reflection space and transmission space with $\mathrm{Si}$ metasurface, respectively (Figure 4g). Due to the resonance occurred when beams propagate back and forth in the nanobrick waveguide caused by an impedance mismatch, the beams with polarization direction along the long axis would be ideally 
reflected, while the polarization direction along the short axis would be ideally transmitted. Corresponding simulation results show $\sim 98.5 \%$ reflection and $\sim 94.3 \%$ transmission of these two orthogonal polarized light with careful design. And the peak response wavelength can be shifted from 1460 to $1625 \mathrm{~nm}$ by simply changing the width of the nanobrick.

\subsection{Polarization-based functional metalenses}

Lenses are widely used in telescopes, microscopes, cameras for imaging or creating designed focuses for lithography or laser cutting. The principle of conventional lens determines its curved shape, therefore they are usually bulky and difficult to fabricate. Metalenses are planar optical elements that can be fabricated using semiconductor processing technology, which can provide an ultrathin alternative to conventional refractive lenses in the future [91, 92]. Nevertheless, dynamically adjusting the properties of lenses, such as focal length, is still difficult, which can only be achieved by mechanically moving the lenses. Fortunately, based on the polarization multiplexing function enabled by polarization and phase co-manipulation principle, it is possible to achieve polarization-dependent switchable multi-focus metalenses. To achieve them, the phase profiles for focuses of different polarization channels should be satisfied with the following formula:

$$
\varphi_{i}(x, y)=k\left({\sqrt{x^{2}+y^{2}+f_{i}}}_{i}^{2}-f_{i}\right), \quad(i=1,2,3 \ldots)
$$

where $k=2 \pi / \lambda$ is wave vector, $\lambda$ is wavelength, $x$ and $y$ are coordinates of metalens plane, and $f$ is the focal length of metalens, respectively. By utilizing the propagation phase, zoom and stereoscopic metalens is proposed by Schonbrun et al. [93]. They encoded two phase patterns into a single array of elliptical nanowires. By this way, they demonstrated two metalenses, one with a different focal length for each linear polarization state and the other with two different optical axes to realize a stereoscopic image. With the geometric phase, Lin et al. [94] designed a two-layer dielectric metalens which has different focus spots for each circularly polarized light as shown in Figure 5a. They also numerically demonstrated a metalens that can control the focal spot position and their relative intensity. However, the multi-layer structure utilizing spatial DoFs will reduce the efficiency and increase the processing difficulty. By combining the propagation phase and geometric phase, Tian et al. [95] proposed a single layer metalens that is switchable between LCP and RCP beams. This metalens can generate two foci along the longitudinal direction as shown in Figure 5b, and their intensity also can be controlled by tuning the ellipticity of incident light. Moreover, they achieved high focusing efficiency of $72 \%$, which was attributed to the optimized design approaches and the low loss of dielectric materials. With the same combination strategy, Gao et al. [96] designed a polarization-selective metalens which establishes three focuses at different positions along the longitudinal direction as shown in Figure $5 c$. The tri-foci metalens was realized by combining two kinds of units which include geometric phase (only change the orientations of nanostructures) and propagation phase (only change the sizes of nanostructures). This method empowers metalens triple functions by simultaneously selecting linear polarization state of incident and emitted light. Moreover, the crosstalk among different focuses is very weak, so it has potential application in multiplexing of optical vortices and high information encryption. Nevertheless, the above strategies are limited to a discrete number of focal points. To achieve better zoom imaging, a continuous tune of the focal length is desirable. Zang et al. [97] proposed a metalens with two focal spots. By changing the orientation angle of the linearly polarized incident light beam at each focal spot, they experimentally demonstrated two focal spots with polarization rotation in the longitudinal and transversal direction, and the schematic of the metalens is shown in Figure 5d. This work realized multiplexing of focusing and rotation and opened a novel avenue to control the light beam. Meanwhile, Maxwell et al. [98] realized a continuous change of focal length from 220 to $550 \mu \mathrm{m}$ with a $40 \mu \mathrm{m}$ diameter metalens. They rotate the linearly polarized input light to impart different phases to different wavelength light, which provides an effective solution for achromatic metalens in a band of the visible spectrum and fuels the development of microscopic imaging. In addition to the axial multi-focus, Figure 5 e shows the splitting and focus abilities of metalens [99], in which multidimensional spin-dependent splitting has been demonstrated by combining geometric phase and dynamic phase. It can simultaneously realize transverse shift and longitudinal focus, and thus can be used in spin control nanophotonics, optical communication, beam shaping and so on.

Resolution is another key merit of metalens. Achieving the highest possible resolution in both imaging and lithography is always a driven force for lens development. Metalens with small pixels allows ultrahigh numerical aperture (NA) for higher resolution and lots of methods have been proposed [100-102]. In addition to increasing NA, vector structured light focusing proved to be capable of 

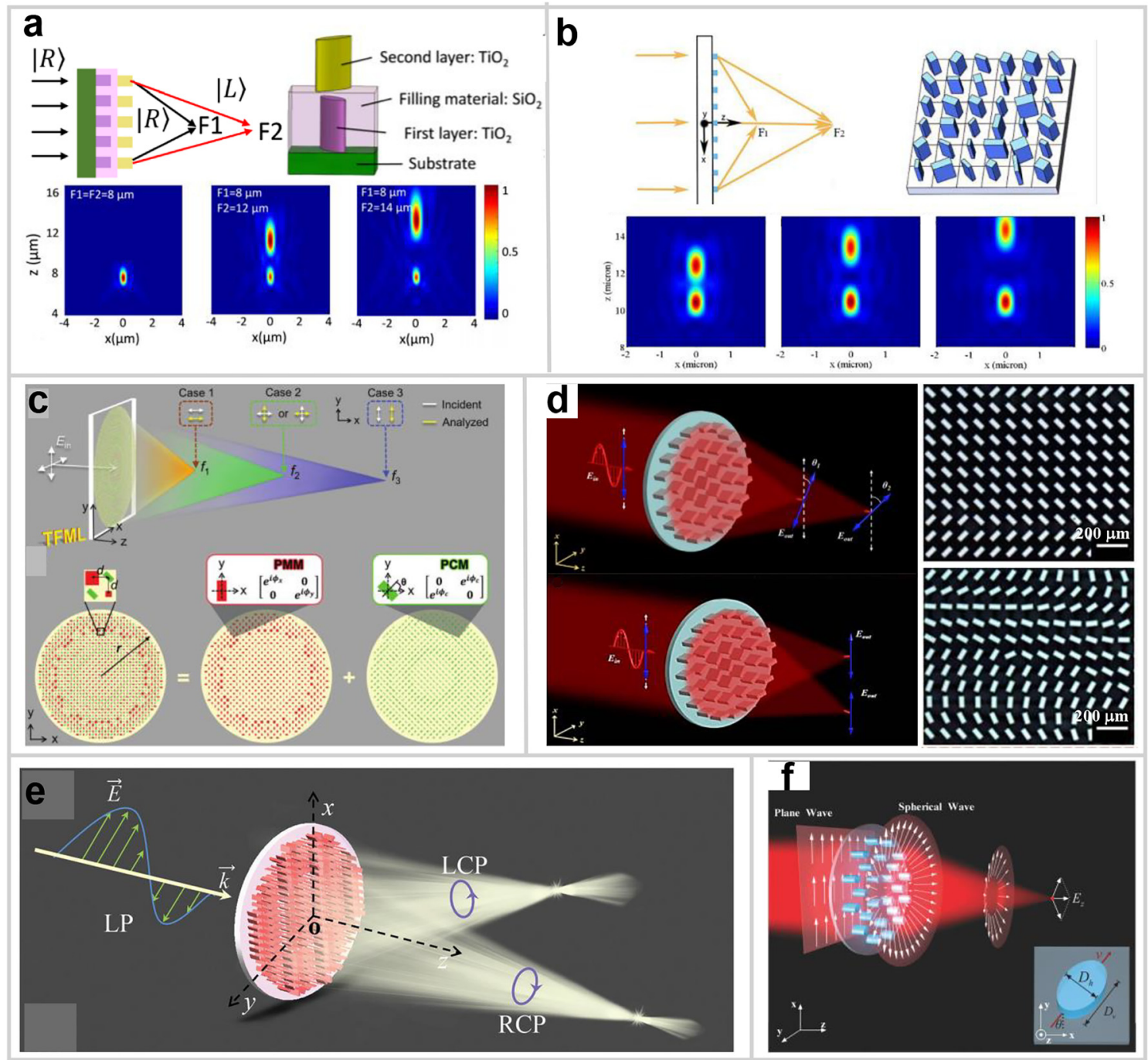

Figure 5: Applications of polarization manipulation in all-dielectric metalenses. (a) Schematics of the two-layer bifocal metalens (left) and schematic of the corresponding 3D configuration (right). $|L\rangle$ and $\mid R>$ represent left circularly polarized light and right circular polarized light respectively [94]. (b) The metalens focuses incident light into two focal spots [95]. (c) Schematic illustration of the polarization-selective trifoci metalens (TFML) [96]. (d) Schematic of the multi-foci metalens with polarization-rotated focal points. Under the illumination of linearly polarized THz waves, there are two longitudinally distributed focal spots. The polarization axis of the incident linearly polarized light beam is rotated at each focal point. The two polarization-rotated angles are $\theta_{1}$ and $\theta_{2}$, respectively [97]. (e) Illustration of the multidimensional manipulation of the metalens, in which transverse and longitudinal spin-dependent splitting are realized from linear polarization to circular polarization [99]. (f) Schematic illustration of designed dielectric metalens illuminated with $x$-polarized light. Inset: Schematic view of the unit cell: $D_{h}$ and $D_{v}$ are diameters of the elliptical cylinder, and rotation angle $\theta$ is the angle between $v$-axis and $y$-axis [104]. Reprint permission obtained from $[94-97,99,104]$.

super-resolution focusing [103]. Using the pixelated polarization control and phase control capabilities of the ADM, Zuo et al. [104] proposed a dielectric metalens that

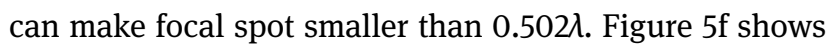
the metalens that can convert linearly polarized incident light to radially polarized light and focus the light simultaneously. Furthermore, they adopt two extra methods to realize sub-resolution, enhancing the longitudinal field component by adding an optimized phase and decreasing the transversely polarized component by filtering the lower spatial frequency.

\subsection{Polarization-dependent multiplexing meta holograms}

Since its invention [105], holography has emerged as a vital technology for shaping wavefront, recording and 
reconstructing real or virtual objects. Due to polarizationinsensitive peculiarity of traditional diffractive optical elements, various holographic multiplexing possibilities are limited. In contrast, metasurfaces can overcome these limitations by employing the polarization-sensitive control with the "polarization plus" schemes, enabling multichannel optical functionalities. Hence, different schemes of polarization multiplexing meta-holograms have been proposed, which extend to amplitude-only holograms, phase-only holograms, dynamic holograms, as well as vector holograms and color holograms, and so forth.

In conventional amplitude-only meta-holograms, the main regulatory parameter is the reflection or transmission amplitude with binary strategy, regardless of the effect of polarization [51, 106]. Desiatov et al. [107] proposed a novel dielectric metasurface to manipulate the phase of incident light, which can reconstruct two different images by switching the circular polarization of illumination in nearinfrared range, as shown in Figure 6a. The metasurface is composed of $\mathrm{Si}$ binary grating with varying orientations to control the geometric phase. Utilizing of the conjugation phase modulation obtained by illuminating with LCP and $\mathrm{RCP}$, a modified Gerchberg-Saxton (G-S) is developed to encode two independent phase profiles for the two orthogonal polarization states. Similarly, Khorasaninejad et al. [108] experimentally demonstrated chirality-dependent phase meta-holograms that project different images depending on the chirality of the incident beam, as shown in Figure $6 \mathrm{~b}$. In this work, two sets of geometric phase nanostructures carry two independent chirality-dependent phase profiles through the interleaved arrangement to realize the switching of two holograms. However, this kind of polarization multiplexing is built on the basis of spatial multiplexing, thus resulting in limited efficiency. Utilizing the propagation phase-based multiplexing schemes in one single meta-atom, Arbabi et al. [67] demonstrated a metasurface that can attain linearly polarization-switchable phase holograms, as shown in Figure 6c. This metasurface exhibited an experimentally measured efficiency up to $97 \%$. However, the above strategies only using geometric phase or propagation phase for polarization multiplexing cannot fully demonstrate the multiple DoF of the meta-atoms. By combing the geometric phase and propagation phase, Mueller et al. demonstrated a $\mathrm{TiO}_{2}$ metasurface which can achieve independent and arbitrary phase profiles on any pair of orthogonal states of polarization [109], as shown in Figure 6d. Fully independent holograms of a "cartoon dog" and a "cartoon cat" on each of any two orthogonal polarizations (including circular and elliptical) are realized by simultaneously changing the orientation angle and dimension of the elliptical meta-atom. Similarly, using the full
DoFs of single asymmetric meta-atom, Zhao et al. proposed a polarization-dependent tri-channel holography by exploring birefringent $\mathrm{Si}$ metasurfaces for the complete control of polarization and phase [110]. Two schemes from orthogonal two channels to twelve channels were comparatively studied. First, two switchable holographic images can be achieved with two linear orthogonal states by encoding two phase profiles into the asymmetric nanofins with different cross-sections but without rotation. Then, by considering the freedom of the entire Jones matrix with three independent structural parameters including length, width and orientation angle of the meta-atom, three sets of independent images can be reconstructed for different combinations of input and output polarization state, as Figure $6 \mathrm{e}$ shown. With these independent three images, twelve different polarization channels and seven independent combinations can be achieved with negligible cross-talk by selecting the combination of input/output polarizations.

Through the co-manipulation of polarization, amplitude and phase, color meta-hologram can be also realized. Wang et al. [111] proposed a strategy to achieve color holograms with interleaved geometric phase metasurface. Three Si meta-atoms with different dimensions for three primary colors are designed based on the wavelength selectivity of CPCE. By placing one red, one green, and two blue units in one pixel, three wavelengthdependent phases for color hologram are realized. Utilizing the same multiplexing schemes and combined the chirality-dependence of the geometric phase, they also demonstrated a polarization-controlled color-tunable holograms as shown in Figure 6f. By encoding the information of the two colors into the two chiral polarized light channels, the color can be tuned by switching the chirality. In the case of elliptically polarized light, the two colors can be merged in different degrees resulting in intermediate colors. Compared to this spatial multiplexing scheme, non-interleaved units are eagerly used to provide high efficiency and resolution. Jin et al. demonstrated a multiplexing metasurface with non-interleaved single-size Si nanofins arrays with 6-bit polarization- and wavelength-dependent encoded color holograms [112]. The geometric phase of the single-sized Si nanofin is utilized to achieve the polarization-dependent tunability which provides the dispersionless phase profile. Based on the dispersion relationship of propagation, two phase profiles are retrieved for RCP and LCP to reconstruct independent red (R), green (G), blue (B) images at a given $z$ plane. Then the two phases are combined together and encoded into a single metasurface according to the geometric phase. By controlling the polarization and wavelength of the incident light, the metasurface is able to 

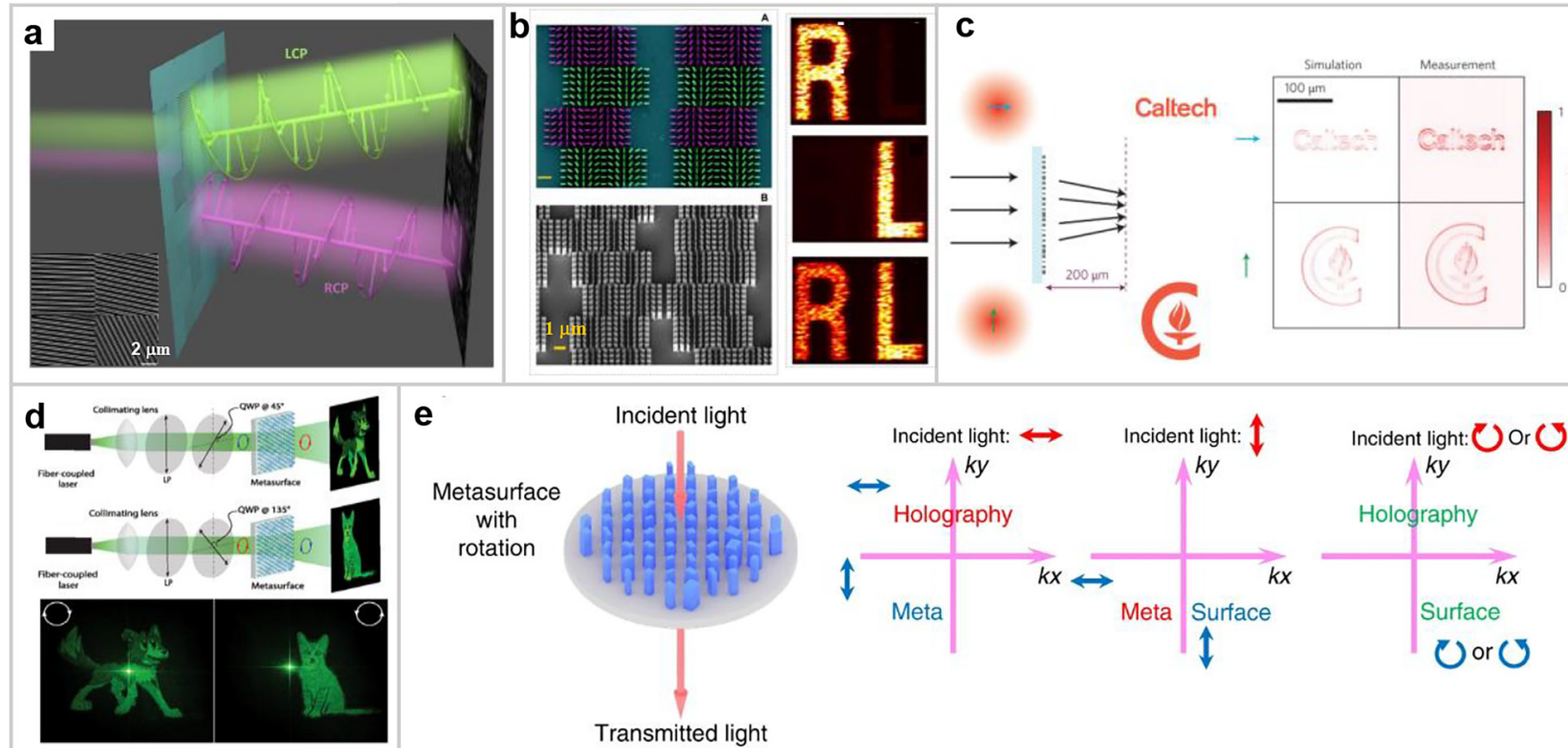

Transmitted light
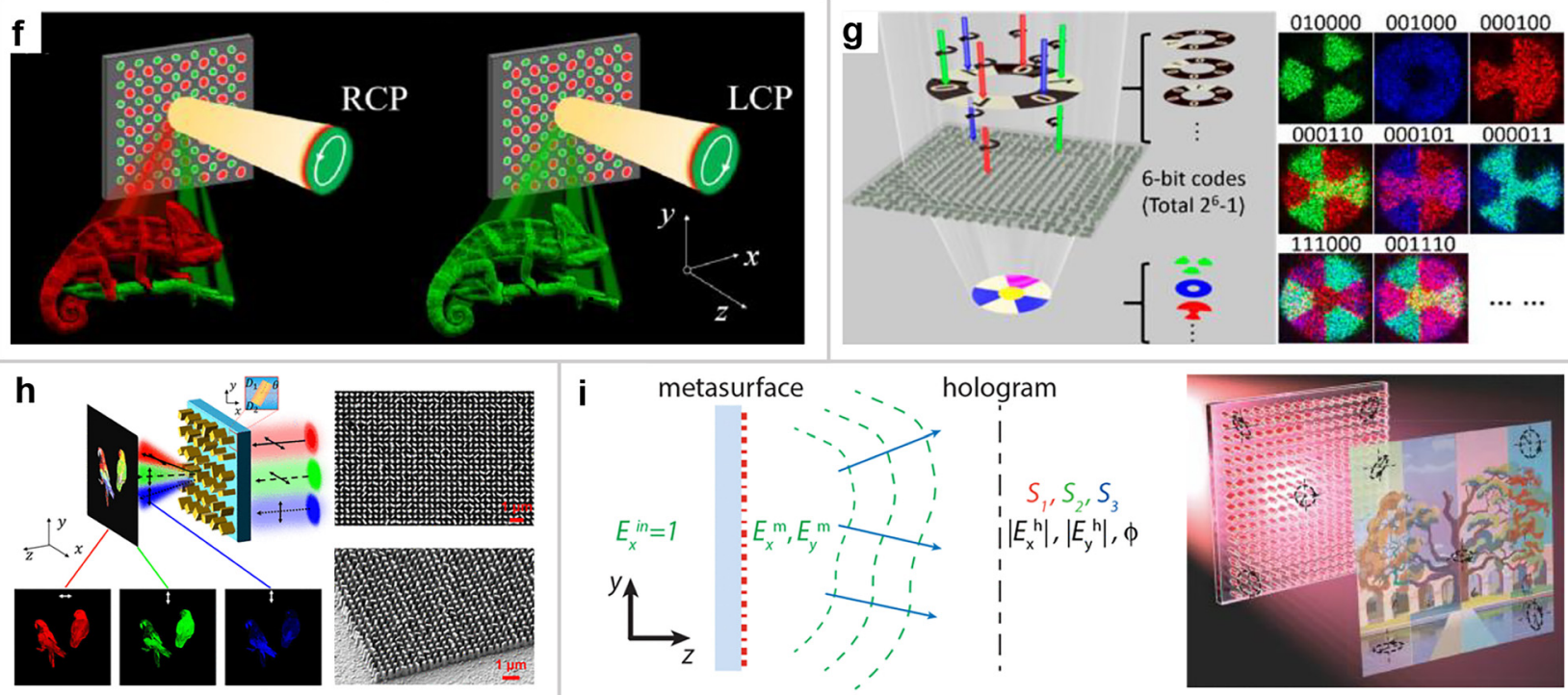

Figure 6: Applications of polarization manipulation in meta-holograms with all-dielectric metasurfaces. (a) A meta-holograms obtained by left and right circularly polarized illumination and the corresponding simulated results [107]. (b) Chirality-dependent phase meta-holograms that project different images depending on the chirality of the incident beam with interleaved meta-atoms design [108]. (c) A polarizationswitchable dielectric metasurface that generates two holograms under the illumination of $x$ - and $y$-polarized light [67]. (d) A TiO ${ }_{2}$ metasurface which can achieve independent and arbitrary phase profiles on any pair of orthogonal states of polarization for fully independent holograms of a "cartoon dog" and a "cartoon cat" [109]. (e) Multi-channel holography by exploring birefringent Si metasurfaces for the complete control of polarization and phase [110]. (f) Schematic illustration of the polarization-controlled interleaved metasurface with different color distributions for RCP and LCP incident polarizations [111]. (g) A multiplexing metasurface with non-interleaved single-size Si nanofins arrays with 6-bit polarization- and wavelength-dependent encoded color holograms [112]. (h) A tri-polarization-channel non-interleaved $\mathrm{TiO}_{2}$ metasurface for far-field color holography with almost zero crosstalk [113]. (i) A vectorial full color hologram with Si metasurface by assigning the red-greenblue data in arbitrary color images to three Stokes parameters [114]. Reprint permission obtained from [67, 107-114].

reconstruct 63 polarization- and wavelength-dependent holographic images, as shown in Figure 6g. But this scheme will bring unwanted images at other locations and can only be used in the Fresnel diffraction zone. $\mathrm{Hu}$ et al. showed a tri-polarization-channel far-field color holography with almost zero crosstalk with noninterleaved $\mathrm{TiO}_{2}$ metasurface [113], as shown in Figure $6 \mathrm{~h}$. By fully using the DoFs of the Jones matrix, three independent phase profiles are encoded into three orthogonal polarization bases with almost zero crosstalk 
for project broadband hologram images. Then trichromatic (RGB) intensity profiles are coupled into the three polarization channels to achieve high-quality and highefficiency vectorial meta-holography in the visible regime. With the similar schemes, Arbabi et al. demonstrated a vectorial full color hologram with Si metasurface. The difference is they assigned the red-green-blue data in arbitrary color images to three Stokes parameters. And then the measured Stokes parameters can be converted back to RGB data to achieve color holograms, as shown in Figure 6i [114].

\subsection{Polarization-based nano-printing}

Nano-printing is an emerging technology in recent years based on the amplitude manipulation of nanostructures such as plasmonic [115] or dielectric antennas [116]. It can achieve ultrahigh-resolution, chemically stable image display, which includes monochrome display and color display. However, nano-printing is usually based on the resonance of nanostructures to achieve wavelength selectivity and the display of color images. As described in section 2.3, the co-manipulation of polarization and amplitude provides a new paradigm for nano-printing design. Dai et al. [117] designed a Si metasurface for high fidelity monochrome grayscale image with a resolution of 84667 dpi (Figure 7a). In their work, asymmetric nanobricks are designed to be a nano-polarizers in reflection mode by carefully optimizing the geometric parameters. Magnetic resonance in dielectric nanobricks is used to suppress the reflectivity of an incident beam with polarization direction along the short axis while letting that along the long axis almost reflected. Combined with Malus's law, by arranging the orientation angle of each nano-polarizer, pixel-level amplitude control can be achieved resulting in continuous grayscale image display. By designing the meta-atoms as a half wave-plates, Zhao et al. [118] also realized hiding the grayscale image in the vector field generated by the Si metasurface (Figure $7 \mathrm{~b}$ ). The nanowaveplates rotate the polarization of the incident light by two times the angle between the incident light and the fast axis. Therefore, complex radial and azimuthal polarization beams can be easily generated. By combing the analyzer at the output end, the intensity of the output field can be continuously tuned for polarization encryption of information. By further utilizing the wavelength selectivity of the geometric phase-based nano-waveplate, Zang et al. [119] demonstrated a metasurface device to encode the color grayscale image into several sets of polarization profiles by controlling the polarization and amplitude as Figure 7c shown. To realize the color image with spatially varying intensity, each element acts as an ultracompact half-waveplate to arbitrarily rotate the polarization direction of light in transmission for a particular color and the analyzer is used to control the amplitude with Malus's law. Two types of nanoblocks with different lateral dimensions are utilized for two primary colors. The conversion efficiency is over $95 \%$ when the resonance is located around $\lambda=660 \mathrm{~nm}$ for Si meta-atoms with $150 \mathrm{~nm}$ length and $105 \mathrm{~nm}$ width. In contrast, the resonance peak is shifted to $\lambda=550 \mathrm{~nm}$ with the structural parameters of $100 \mathrm{~nm}$ length and $75 \mathrm{~nm}$ width and the corresponding conversion efficiency is $80 \%$. Actually, based on cosine square in the Malus's law, there are four orientation angles for the polarizer in the range of $[0, \pi]$ to generate an equal intensity, which provides a new degree of design freedom. Utilizing this principle, Deng et al. proposed a dualchannel polarization-dependent metasurface for nanoprinting display composed of the single-sized Si metaatoms [120]. By utilizing four orientation candidates to generate an equal intensity, two of the four orientations can be chosen to encode an extra binary image into a single metasurface after rotating the sample by $\pi / 8$. Figure $7 d$ shows the method to decode two meta-images and the corresponding experimental results. Applying different resonance response of the asymmetric structure along the orthogonal axes to achieve multiple wavelength amplitude control in orthogonal linearly polarized directions, Yang et al. [121] demonstrated polarization-dependent structural color in the reflection mode in the visible range by utilizing $\mathrm{TiO}_{2}$ elliptic meta-atoms (Figure 7e). In the work, the phenomenon of Fano resonance was also utilized to achieve sharp contrast of colors under two orthogonal linear polarizations by varying the periods of the meta-atoms along the $x$-axis and $y$-axis.

\subsection{Polarization-based multifunctional devices}

Conventional refractive or diffractive elements or general metasurface devices are designed for a single function, such as beam deflection, lens, holography, nano-printing as described above. Fortunately, the capabilities of polarization-dependent multiplexing and the comanipulation of polarization, phase and amplitude can realize the integration of different functions in one single ultra-compact ADM-based device. Gao et al. [122] proposed and experimentally demonstrated a polarization- 

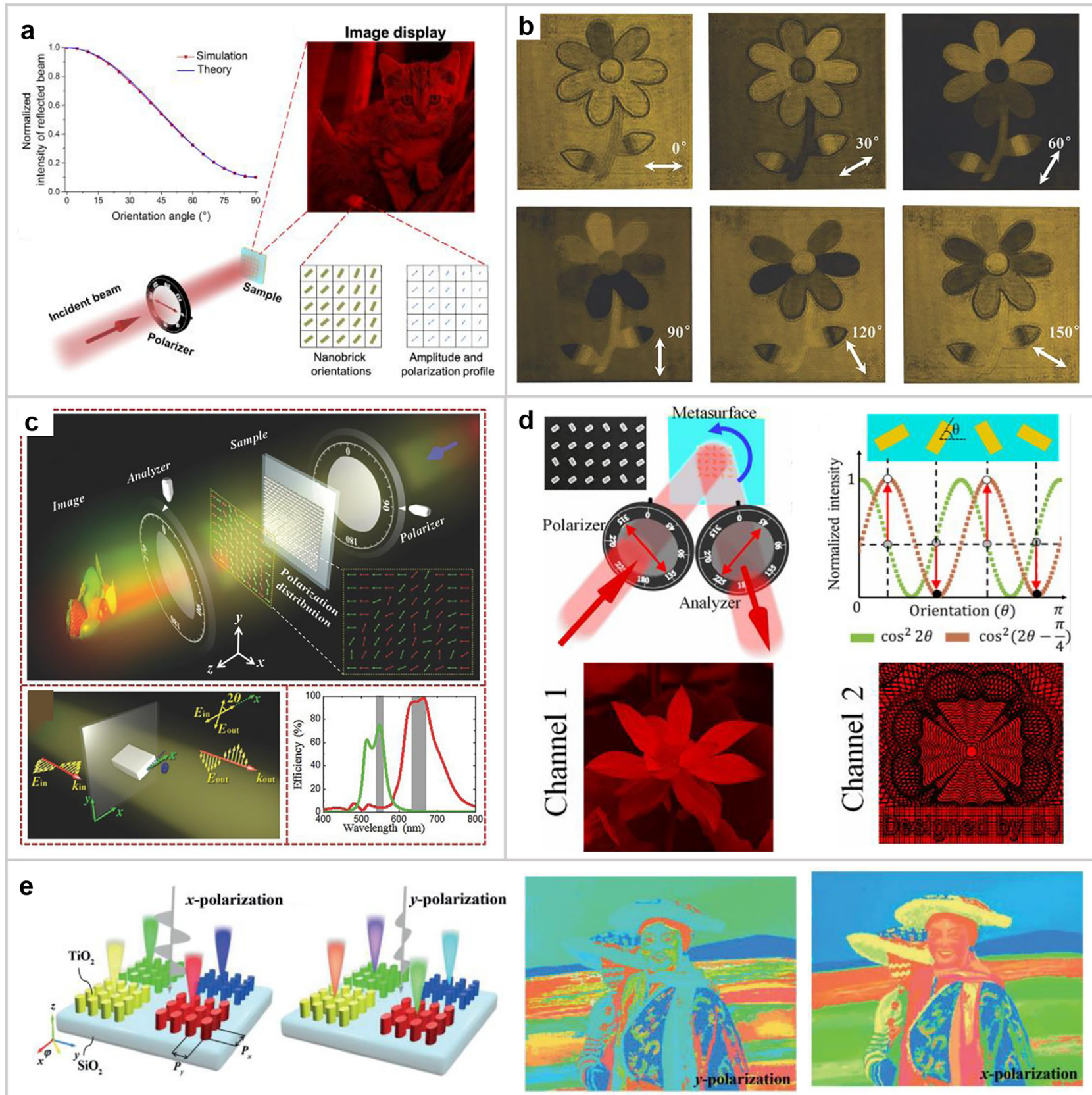

Figure 7: Applications of polarization-based nano-printing with all-dielectric metasurfaces. A Si metasurface that realizes the gray-scale image display, where the meta-atoms inside act as (a) polarizers [117] or (b) half waveplates by combing the analyzer at the output end [118]. (c) A metasurface device to encode the color grayscale image into several sets of polarization profiles utilizing the wavelength selectivity of the geometric phase elements [119]. (d) A dual-channel polarization-dependent metasurface for nano-printing display composed of the singlesized $\mathrm{Si}$ meta-atoms [120]. (e) $\mathrm{A} \mathrm{TiO}_{2}$ metasurface for polarization-dependent structural color in the reffection mode in the visible range applying different resonance response of the asymmetric structure along the orthogonal axes [121]. Reprint permission obtained from [117-121].

switchable bi-functional Si metasurface for anomalous beam deflection and focusing in the visible band as shown in Figure 8a. A rectangular meta-atom is used to design two distinct propagation phase profiles for beam deflection and focusing along the fast and short axes by changing its length and width. By tailoring the periodicity of the metaatom, the beam deflection angle and focusing distance can be efficiently tuned. By further utilizing the ability of multiparameter control of polarization, amplitude, and phase by ADM, researchers have explored integrating more 
functions into one device, where the combination of nanoprinting and holography is a highlight [123]. Zhang et al. utilized the simple interleaved geometric phase metaatoms to realize the multiplexing of nano-printing and holography (Figure 8b). In the design, the two groups of meta-atoms that realize the hologram and the polarization distribution containing the grayscale information of the image are arranged interleaved. In addition, due to the chiral conjugation of the geometric phase, holographic polarization switching can also be achieved. Yoon et al. [124] presented a dual-mode geometric phase-based Si metasurfaces that enable control of phase and spectral responses simultaneous for color printing in reflection mode and holography in transmission mode (Figure 8c). Different from the single asymmetric geometric phase structure, parallel double-nanorod is used here to increase the CPCE for the geometric phase. Two such meta-atoms with different sizes are optimized to achieve the same CPCE at the wavelength of $635 \mathrm{~nm}$ and two distinct reflection responses, which makes the information of color printing and hologram uncoupled from each other. By this way, the device shows a reflective display under white light illumination but generates a transmissive far-field hologram that reconstructs the phase information under singlewavelength coherent light illumination. However, this single wavelength geometric phase design also makes the hologram only monochromatic. And it is difficult to achieve grayscale amplitude control of nano-printings. To address these problems, on one hand, Wei et al. [125] achieved a two-color nano-printing and holography in one Si metasurface by designing two geometric phase-based nanostructures with wavelength-sensitive CPCE as shown in Figure 8d. Si dimers and nanofins are chosen to achieve a reasonably good wavelength selectivity with CPCE of about $20 \%$ and $50 \%$ at the $540 \mathrm{~nm}$ and $645 \mathrm{~nm}$. Since the nanostructure needs both control wavelength amplitude and the phase, the distribution of the holographic phase and the arrangement of color printing are coupled with each other. To solve the problem, a modified parallel iterative Gerchberg-Saxton algorithm is developed to obtain holograms with arbitrary pixel arrangements. On the other hand, Overvig et al. [126] presented a Si metasurface with the capability of complete amplitude and phase control for monochromatic grayscale nano-printing and far-field holography (Figure 8e). In the design, geometric phase and propagation phase are combined by varying the sizes, which determine the degree of birefringence, and rotation angles. By this way, the incident CP light can be converted into any polarization state. With the polarization filter for only selecting the cross-polarization components, the amplitude can be continuously controlled by the CPCE, while the phase is a sum of the propagation phase and the geometric phase. Finally, combining the characteristics of the above work, Bao et al. [127] demonstrated crystal Si metasurfaces to simultaneously realize nano-printing with continuous HSB color tuning and full-color holography (Figure 8f). Three nanoblocks with different sizes and high aspect ratio are first designed for displaying three primary colors by utilizing the wavelength selectivity of the CPCE. Then a double-nanoblock cell composed of two nanoblocks with identical size but different orientation angles is applied to continuously control the density by tuning the rotation angle difference between the two nanoblocks. In addition, the phase can be independently controlled by the orientation angle of one of the nanobricks in the doublenanoblok cell. Therefore, if three double-nanoblok cells with the sizes of the three primary color are put in one pixel, continuous HSB color control can be realized by continuous intensity tuning of the three primary colors. Meanwhile, with algorithm design, two sets of information of images are decoupled and displayed with color nanoprinting and holography in one single metasurface.

\subsection{Polarization detection}

Since the polarization of the reflected or transmitted light of the object contains rich information that is cannot be obtained from the intensity, so the polarization detection has important applications in the field of film thickness measurement, remote sensing, machine vision and so on. Traditional spatial light path measurement requires discrete optical components, which results in a complex system and requires multiple measurements. The metasurface has a unique polarization control capability and multi-functional integration capabilities, making it an attractive platform for polarization detection. And because of its features of ultra-light and ultra-compact, it is expected to achieve a more integrated optical system.

Since the Stokes parameters can be used to calculate the polarization by light intensity, make the measurement of them is an effective means to evaluate the SOP of the incident light. Wei et al. [128] proposed a dielectric metasurface composed of elliptical Si pillars, which acts not only as a polarization separator but also a flat focusing lens. The metasurface is composed of pixels, which is composed of four sub-pixels designed to focus one of the $x-, y-, 45^{\circ}$ linear polarized light and left circular polarized light, respectively. Therefore, the intensities of the basic polarization components $\left(I_{x}, I_{y}, I_{a}, I_{l}\right)$ in the incident light can be immediately determined in four areas in one measurement to get the 


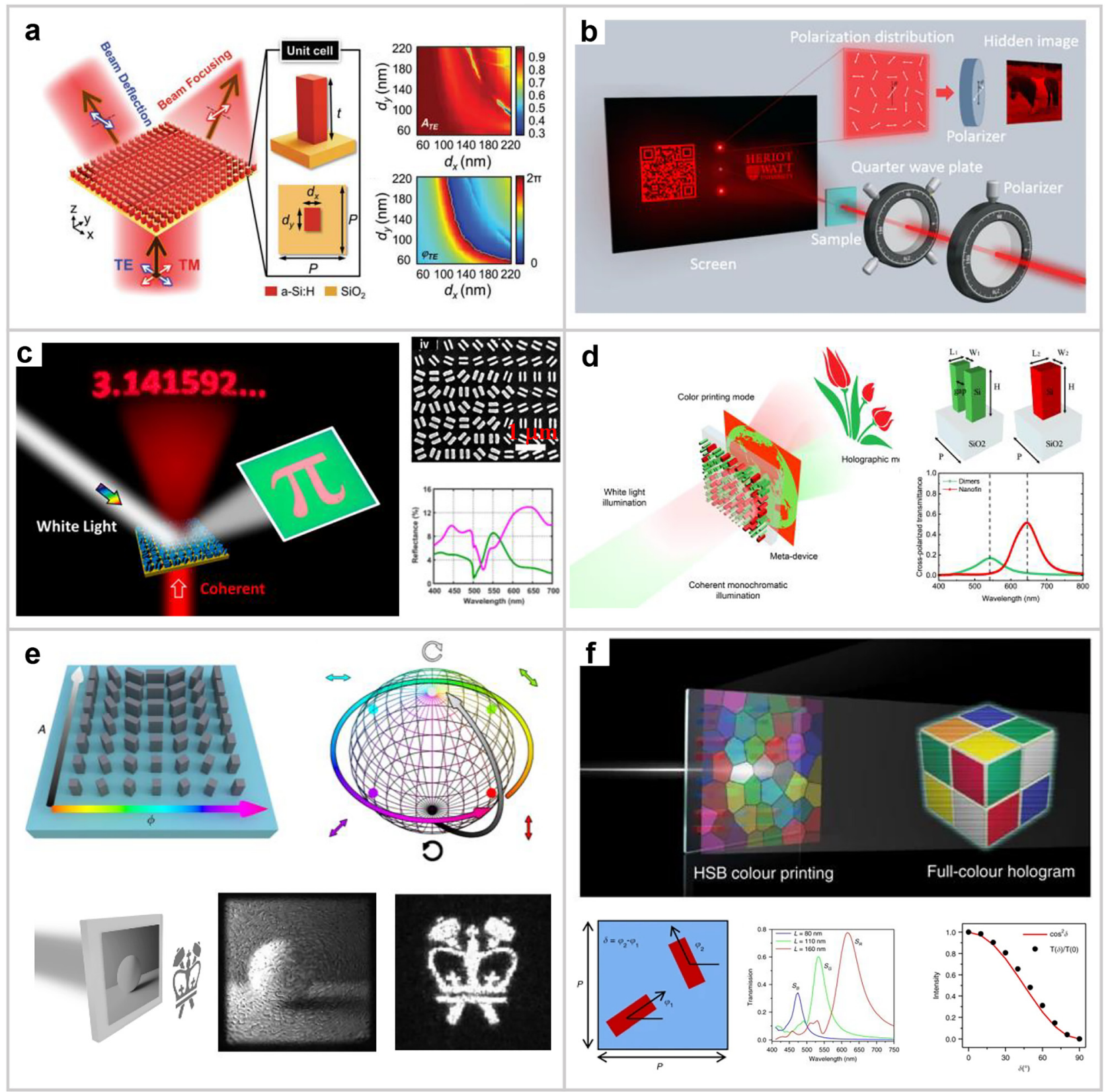

Figure 8: Applications of polarization-based multifunctional devices with all-dielectric metasurfaces. (a) A linear polarization-switchable bifunctional metasurface for anomalous beam deflection and focusing in the visible band based on propagation phase [122]. (b) Multiplexing of nano-printing and chirality-dependent holography with interleaved geometric phase meta-atoms [123]. (c) A dual-mode geometric phasebased Si metasurfaces that enable control of phase and spectral responses simultaneous for color printing in reflection mode and holography in transmission mode [124]. (d) A two-color nano-printing and holography in one Si metasurface by designing two geometric phase-based nanostructures with wavelength-sensitive polarization conversion efficiency [125]. (e) A Si metasurface with the capability of complete amplitude and phase control for monochromatic grayscale nano-printing and far-field holography [126]. (f) A crystal Si metasurface to simultaneously realize nano-printing with continuous HSB color tuning and full-color holography [127]. Reprint permission obtained from [122-127].

Stokes parameters, as shown in Figure 9a. Similarly, Guo et al. [129] designed a GaN metasurface, in which the same four basic polarization components as above can be focused simultaneously on symmetrical positions in space. The difference is that they only use three sub-pixels to achieve this function, since $x-, y$ - polarization focus can be achieved using the propagation phase multiplexing schemes. In order to obtain the intensities of the six basic polarization 
components, Arbabi et al. [130] proposed six-domain Si metasurfaces to get full Stokes characterization of the polarization state by splitting and focusing light using three different sub-pixels, as shown in Figure 9b. Each sub-pixel can focus a set of orthogonal polarizations to two positions off-axis, respectively, utilizing the polarization multiplexing design. With this approach, they also created a metasurface mask for polarizing images, demonstrating the ability to make polarizing cameras. Also using the measurement of six basic components, Yang et al. [131] demonstrate a new type of Hartmann-Shack sensor with metalens array as shown in Figure 9c. This device can not only measures polarization profiles but also phase profiles presents by inspecting the foci amplitudes, which can be applied to measure partially polarized beams according to the feature of the stokes parameter. Different from the way of Stokes parameters measurement, Zhang et al. [132] employed a multiplexed geometric phase meta-hologram to directly achieve polarization detection. The intensity ratio of two points reflects the amplitude ratio of the circularly polarized orthogonal basis and the orthogonal basis phase difference can be the directly observed the azimuth angle of the dark area owing to the interference of a spatially varying phase difference. This is an ingenious design but the efficiency is only less than $1 \%$ due to the optical absobtion, which can be greatly improved by using the dielectric metasurface.

Another aspect of polarization detection is direct polarization imaging combined with the lens, which can be widely used in pattern recognition, biological detection and other fields. Khorasaninejad et al. [133] proposed a planar metalens consisting of interleaved $\mathrm{TiO}_{2}$ nanorods, which can form two focuses of the opposite charity at the same time, as shown in Figure 9d. Each group in the interleaved nanorods generates an off-axis focus with same focal length for light of LCP and RCP. By this way, two images can be formed of an object in the same field of view and opposite charity. Due to the non-dispersive nature of the geometric phase, they also proved multi-spectral

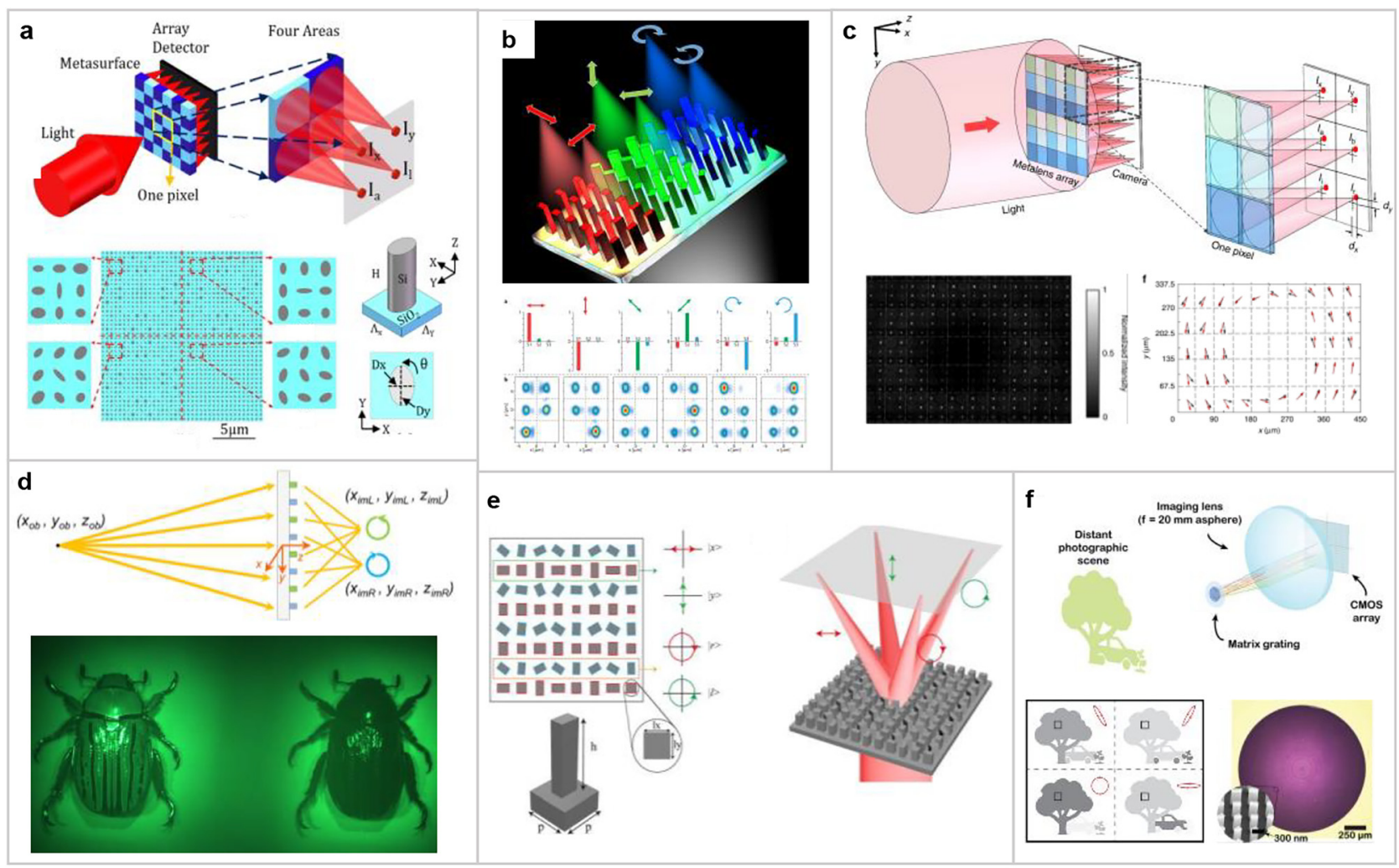

Figure 9: Applications of polarization detection with all-dielectric metasurfaces. (a) A Si metasurface-based polarimeter array for Stoke parameters detection by measuring $x-, y-, 45^{\circ}$ linear polarized light and left circular polarized light [128]. (b) A Si metasurface dividing light into six basic polarization components for Stoke parameters measurement [130]. (c) A Si metasurface-based generalized Hartmann-Shack sensor for state of polarization and phase profile measurement [131]. (d) $A \mathrm{TiO}_{2}$ metasurface forming two images of an object in the same field of view and opposite charity [133]. (e) $\mathrm{A} \mathrm{TiO}_{2}$ metasurface focusing four kinds of polarizations to four spots for both linear polarization imaging and chiral imaging [134]. (f) A metasurface-based grating for full-Stokes polarization analysis [135]. Reprint permission obtained from [128, 130, 131, 133-135]. 
polarization imaging. In addition of chiral Imaging, Yan et al. [134] proposed an all-Si metasurface that forms images simultaneously in two orthogonally polarized groups in real time, and realizes linear polarization imaging and chiral imaging, as shown in Figure 5e. It is interesting to note that this metasurface is based on a staggered configuration rather than a segmented configuration, so there is no need to align the incident light exactly. To achieve a full-Stokes polarization imaging, Rubin et al. designed $\mathrm{TiO}_{2}$ metasurface-based matrix gratings for arbitrary parallel polarization analysis as shown in Figure 9f [135]. The matrix Fourier optics is used to describe optical elements that can generate polarization dependent functions in their diffraction orders. They applied this principle to design periodic gratings to analyze arbitrary polarizations in parallel, enabling a polarization camera with no additional polarization optics, moving parts, or specialized sensors. Practical polarization photography with this camera was demonstrated, which takes the metasurface polarization detection one step further to the real applications.

\subsection{Vector vortex beam generators}

Vector beams and vortex beams have plenty of applications due to their characteristic optical properties [136]. These two types of beams are also known as exotic beams. Among them, vector beams possess exotic polarization (spatially varying polarization distribution), and vortex beams possess exotic phase (helical phase wavefront). The beams which simultaneously possess the characteristics of both of them are called vector vertex beams (VVB). They are widely applied in particle trapping, optical imaging, communication and quantum optics. As described in Section 2.3, dielectric metasurfaces are efficient and miniaturized devices to achieve spatial arbitrary polarization distribution because of its ability of pixelated polarization and phase co-manipulation which can be applied to generate vector vortex beams. The generation of vector beams with ADMs has been theoretically and experimentally demonstrated by Chen et al. [137]. They designed dielectric metasurfaces which can transform linearly polarized light into vector beams. The metasurface is fabricated by femtosecond laser etching in glass, each cell acts as a half-waveplate and the direction of the slow axis varies with the azimuth angle of the metasurface, as shown in Figure 10a. Therefore, according to the geometric phase principle, three metasurfaces with different distribution of slow axis can generate three types of vector beams with different polarization orders and phase orders. As a result, the coexist of vector beams and vortex beams is proved. After that, He et al. proved the dielectric metasurface not only can generate vortex and vector beams, but also can switch between them [138]. The schematic of the optical system is shown in Figure 10b. Generation of vortex beam and vector beam can be understood easily, the quarterwaveplate was used to connect the two processes. In this work, the conversion efficiency of the vector beam to vortex beam is as high as $\mathbf{4 7 . 7 \%}$. It shows that the metasurface has the ability to control the polarization flexibly. Figure 10c shows a metasurface can generate multiple vectorial vortices, which proposed by Maguid et al. [139]. They demonstrated multifunctional multiple beam technology, and the resultant beams from linearly polarized light through the interleaved geometric phase dielectric metasurface are axially symmetric superposition of vortices with opposite helicities. So rotation angle of incident linearly polarized light according to the fringes of the intensity pattern can be obtained. Though metasurface possesses excellent performance in controlling VVB, the function cannot be changed once it is fabricated. Wang et al. [140] realized an arbitrary generation of VVB on the hybrid-order Poincare sphere, in which they integrated liquid crystal with dielectric metasurface, as shown in Figure 10d. By changing the phase of liquid crystal via controlling the voltage, desirable VVB can be generated. In all the above schemes of VVB generation, the annular intensity profiles of the VVB vary with their topological charges, which may cause problems when compositing VVBs because the intensity profiles of different VVBs cannot closely coincide with each other. To tackle this problem, Liu and his co-workers proposed a new method to generate the perfect vector vortex beam [141], and the schematic is shown in Figure 10e. The system consists of vector plate, Bessel converter and lens, they are all designed by computing the relationship between the optical axes and polarization of incident light. To further miniaturize the device for applications in integrated optical systems, Qiu et al. [142] proposed a single layer metasurface for generating perfect vector vortex beams very recently. The metasurface consists of two identical nanoposts with varied positions and rotated angles, as shown in Figure 10f. Therefore, there are four parameters that can be manipulated in this design, importantly, they found it is enough to achieve full control of parameters of light (includes amplitude, phase and polarization) by using genetic algorithm and vector diffraction theory. Then they generated perfect vector vortex beams, which experimentally confirmed that metasurface can realize independent and arbitrary control of the amplitude, phase and polarization of light. 


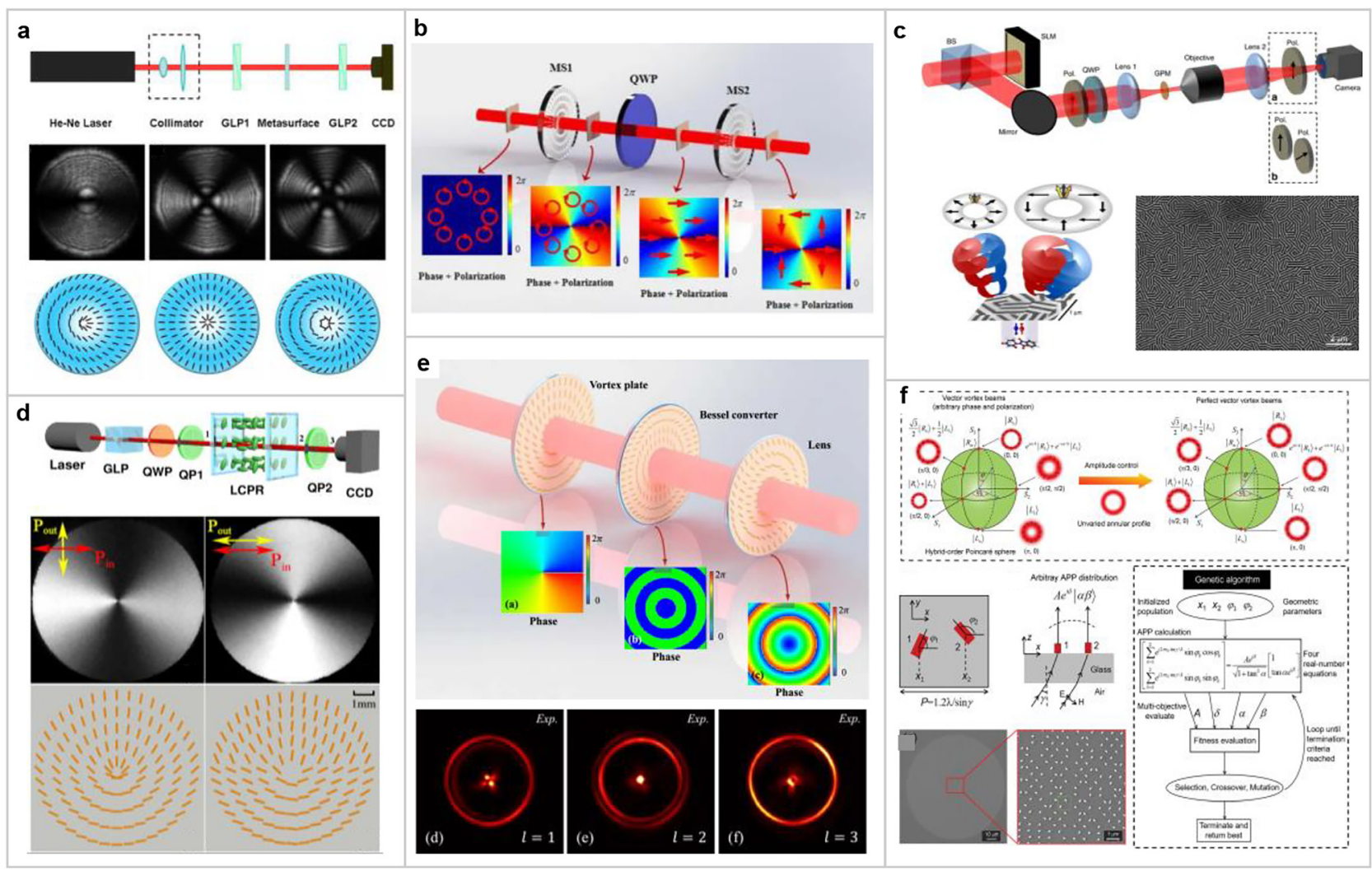

Figure 10: Applications of vector vortex beam generators with all-dielectric metasurfaces. (a) The pattern of three types of output vector beam through the polarizers (up), the corresponding dielectric metasurfaces with polarization order $q=0.5,1.0,1.5$, respectively (down). The black lines represent the slow axis spatial distribution of the metasurfaces [137]. (b) The schematic of the vector vortex beam generation, in which MS means metasurface and QWP means quarter-waveplate [138]. (c) Schematic of vectorial vortex beams with winding numbers $l=1$ and $l=2$ emitting from a metasurface illuminated with linearly polarized light; red and blue helices represent vortices with opposite helicities [139]. (d) The optic system to generate vector vortex beams. GLP: Glan laser polarizer; QWP: quarter-waveplate; QP: metasurface [140]. (e) The optical element system used to generate perfect vortex beams. Insets indicate the phase wavefront at the element plane [141]. (f) Top and side views of a unit of metasurface under $y$-polarized light at oblique incidence angle $\gamma \cdot A e^{i \delta} \mid \alpha \beta>$ represent amplitude, phase and polarization of the output light [142]. Reprint permission obtained from [137-142].

\subsection{Quantum optics}

It is known that the angular momentum of light has vitally huge potential for realizing high dimensional quantum spaces to each photon [143]. Converting the SAM of light into OAM of light is a typical method [144-146] to connecting these two kinds of angular momentums, in which polarization manipulation plays an essential role. Recently, dielectric metasurface has been introduced as a novel device for quantum optics because geometric phase elements provide a natural connection to directly connect optical SAM with OAM [147]. For example, by using dielectric metasurfaces [146], consisting of the same elements with spatially varying orientation, circular polarization can be converted to state with opposite spin and extra OAM. However, because only the elements' orientation angles vary, the output OAM states are not independent which constrained to be values of $\pm 2 a \hbar$ ( $a$ is a constant). In addition, the SAM is limited to circular polarizations. Figure 11a shows the schematic of the concept proposed by Devlin et al. for arbitrarily converting SAM to OAM with dielectric metasurface [145]. This metasurface creates vortex beams whose handedness is determined by the circular SOP of the incident light. The arbitrary spin-toorbital angular momentum conversion of light is realized by superposing LCP and RCP states with independent values of OAM. In addition, according to Chen and coworkers' work [144], they can generate OAM with high efficiency for visible light that wavelength region ranging from 635 to $730 \mathrm{~nm}$, and the control of the SOP of incident light makes OAM multicasting possible.

With the capability of polarization multiplexing enabled by polarization and phase control, multi-photon manipulation can be realized. Stav et al. [148] 


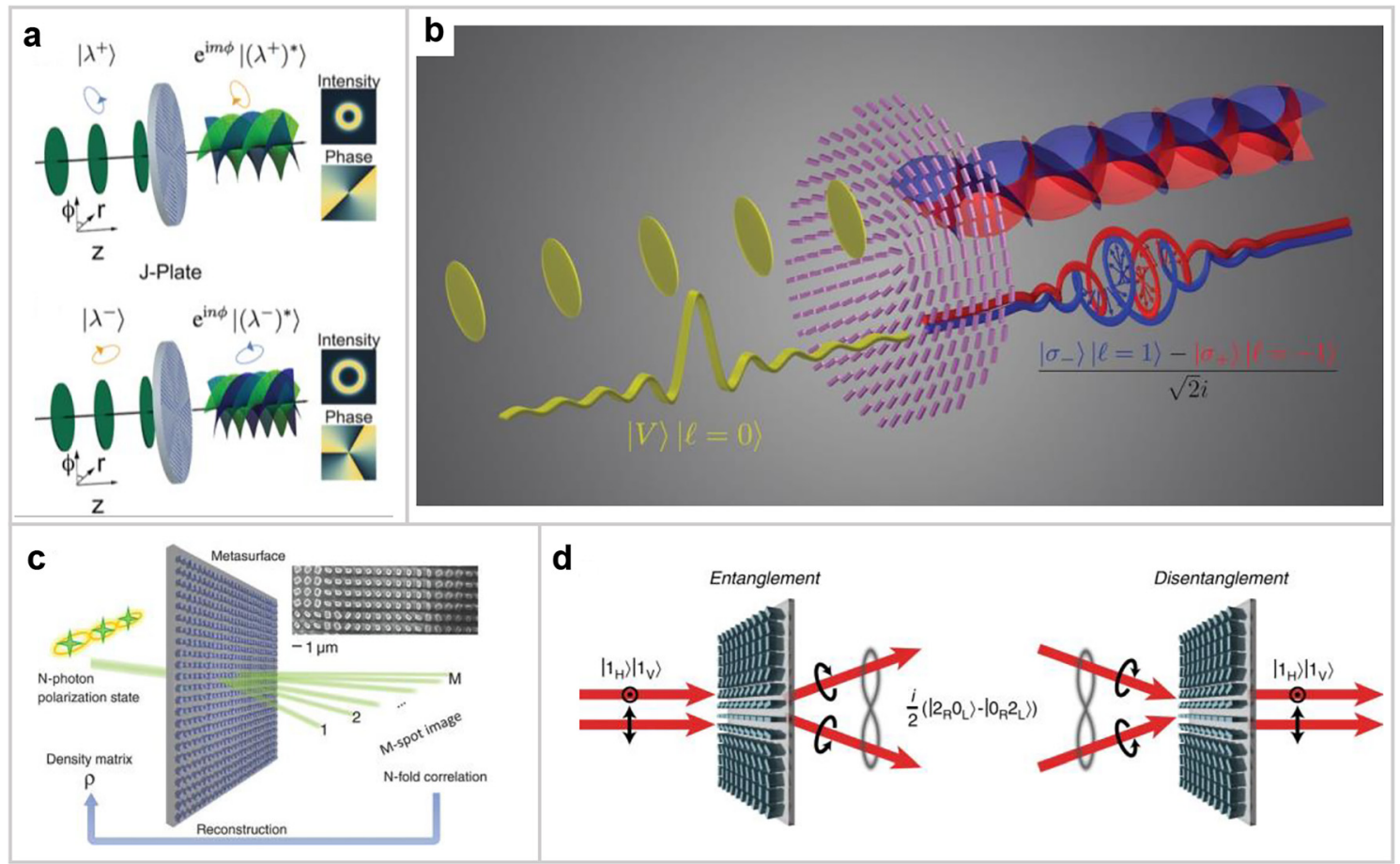

Figure 11: Applications of in quantum optics with all-dielectric metasurfaces. (a) Schematic of the left and right circularly polarized light converted into vortex light [145]. (b) Schematic of metasurface to form entanglement between spin and OAM on a single photon, yellow plane represents plane wavefront, red and blue represent opposite vortex phase wavefront [148]. (c) The schematic of metasurface which can make $\mathrm{N}$-photon image at M-spot. Insertion is a scanning electron microscopy image of all-dielectric metasurface [149]. (d) Schematic of metasurface that used to generate entanglement and disentanglement of two photons state. Entanglelment: two photons with orthogonoal linear polarization state are divided into circular polarization components in the same channel (left). Disentangle: The inverse of the above process (right) [150]. Reprint permission obtained from [145, 148-150].

demonstrated a dielectric metasurface can generate quantum entanglement of the spin and orbital angular momentum of photons. The schematic of the metasurface is shown in Figure 11b. This is achieved by using $\mathrm{Si}$ based geometric phase metasurface with a photonic spin-orbit interaction mechanism as described above. Although the principle is relatively simple, generation and manipulation of entangled photon states with metasurface open new avenues for nanophotonic quantum information applications. To realize the manipulation of multiple quantum states, Wang et al. [149] proposed a dielectric metasurface, which can reconstruct amplitude, phase, coherence, and entanglement of multiphoton polarization-encoded states simultaneously, as shown in Figure 11c. This approach is suitable for measuring SOP in quantum imaging. As a result, metasurfaces may become a powerful tool for future quantum optics and may be used extensively in photonic quantum information systems. As to the specific application in quantum optics, metasurfaces can provide advanced solutions for quantum imaging, sensing and computing. Georgi et al. [150] proposed a metasurface interferometry toward quantum sensors. Figure $11 \mathrm{~d}$ shows entanglement and disentanglement of two-photon states using an ADM. Based on the geometric phase metasurface, two photons with orthogonal linear polarization can be divide into two circular polarization channel, but this quantum state cannot become two single photo states due to its NOON state, thus quantum entanglement happened, furthermore, disentanglement also can be realized via quantum interference of the metasurface. Above examples indicate that metasurface is suited to implement large scale and high dimensional quantum manipulation.

\section{Conclusions and outlook}

Metasurface with the ability of multi-parameter manipulation to arbitrarily construct the near-field and far-field light wave is an attractive platform for fundamental and applied researches. Compared to plasmonic metasurface, ADMs composed of high-refractive-index dielectric materials can achieve extraordinary transmission efficiency. Combining with the CMOS compatible processing technology, the ADMs are highly promising to form ultra-thin, 
ultra-compact and multifunctional optical systems for practical applications. Pixelated arbitrary polarization manipulation at subwavelength is a unique advantage of metasurfaces and it also provides a link between the classical optics and quantum optics. Through reviewing recent works on polarization manipulation based on ADMs, we have summarized the principles and schemes of metasurface-enabled polarization control. A "polarization plus" framework is summarized to include almost the all previously exciting researches related to polarization by ADMs. Based on this framework, various polarization-based applications ranging from polarization convectors, beam splitters, metalenses and meta-holograms, polarization detection and imaging to vector vortex beam generators and quantum optics are introduced.

We believe that there will be rapidly increasing achievements emerge with further using the polarization manipulation capability of ADMs. For example, ADMs with the function of polarization detection or imaging can be easily integrated with the image sensor to achieve an ultra-compact on-chip integrated polarimeters or polarization imaging system without involving bulky separate polarization optics and moving parts, which can be widely applied in areas such as remote sensing, machine vision, biomedical imaging and diagnosis, and material analysis. Besides, by combining the polarization manipulation with other more parameters manipulation mechanisms, more complex multi-functional metasurfaces can be realized, such as achromatic polarized multi-focus lenses, nonlinear polarization metasurface and metasurfaces with more channels. Furthermore, arbitrary vector beams enabled by pixelated polarization conversion still have a lot of space for fundamental and applied researches, such as vector holography, vector color printing used for data encryption and storage, polarization optimization in super-resolution focusing and more complex vector vortex beams in quantum sensing. In addition, by utilizing smart materials, dynamically adjustable polarization control can be achieved, such as the electrically tunable polarization control may be realized by combining with the birefringence characteristics of liquid crystal.

Although polarization-controlled ADMs have shown a promising future, there are still some challenges that need to be addressed to push them for real applications. For example, to achieve more complex metasurfaces for multitasks, the coupling mechanisms of multi-parameter regulation are still not much clear, which need further studies. Also, when designing the polarization multifunctional metasurfaces, it is hard to consider the performance of the devices such as efficiency or aberration, which requires multi-objective optimization. Recently emerged physical-driven [151] or data-driven inverse design [152, 153] for nanophotonic devices may be a possible solution.
From the materials perspective, there is rare research on the metasurface in the ultraviolet band at present, and it is necessary to find suitable materials platform such as $\mathrm{HfO}_{2}$, $\mathrm{Ta}_{2} \mathrm{O}_{5}, \mathrm{Nb}_{2} \mathrm{O}_{5}$ to utilize the polarization manipulation ability in the ultraviolet band. And more dielectric materials such as $\mathrm{ZrO}_{2}$ to satisfy extreme conditions such as high temperature, high pressure, and high power should be explored. Finally, the manufacturability of metasurface has to be considered. Most current dielectric metasurfaces are experimentally demonstrated utilizing electron beam lithography (EBL) method. However, EBL-based process is too costly and inefficient for the real products. Recently, there are some attempts to use CMOS processes for large area fabrication of metasurfaces [154-156]. Besides, the combination of EBL and nanoimprinting may be another high-throughput and lower cost processes for highly customizable optical components. In the near future, it is foreseeable that more researchers enter this field to promote further development of ADMs with polarization control, and more researches and even commercial products will emerge.

Acknowledgments: This work was supported by the National Natural Science Foundation of China under contract numbers 51722503, 51621004, and Natural Science Foundation of Hunan Province of China under contract number 2020JJ5059. The project is also supported in part by Science and Technology Bureau Foundation of Changsha City under contract number kh1904005 and Youth Innovation Promotion Association of the Chinese Academy of Sciences under contract number No. 2017429.

Author contribution: All the authors have accepted responsibility for the entire content of this submitted manuscript and approved submission.

Research funding: This work was supported by the National Natural Science Foundation of China under contract numbers 51722503, 51621004, and Natural Science Foundation of Hunan Province of China under contract number 2020JJ5059. The project is also supported in part by Science and Technology Bureau Foundation of Changsha City under contract number kh1904005 and Youth Innovation Promotion Association of the Chinese Academy of Sciences under contract number No. 2017429. Conflict of interest statement: The authors declare no conflicts of interest regarding this article.

\section{References}

[1] V. G. Veselago, "The electrodynamics of substances with simultaneously negative values of $\varepsilon$ and $\mu$," Sov. Phys. Uspekhi, vol. 4, pp. 509-514, 1968. 
[2] J. B. Pendry, A. J. Holden, D. J. Robbins, et al., "Magnetism from conductors and enhanced nonlinear phenomena," IEEE Trans. Microw. Theory, vol. 47, pp. 2075-2084, 1999.

[3] W. J. Padilla, D. N. Basov, and D. R. Smith, "Negative refractive index metamaterials," Mater. Today, vol. 7-8, pp. 18-35, 2006.

[4] B. Vasić, G. Isić, R. Gajić, et al., "Controlling electromagnetic fields with graded photonic crystals in metamaterial regime," Opt Express, vol. 18, p. 20321, 2010.

[5] J. D. Joannopoulos, P. R. Villeneuve, and S. Fan, "Photonic crystals," Solid State Commun., vol. 2-3, pp. 165-173, 1997.

[6] L. Ran, J. Huangfu, X. Zhang, et al., "Left-handed materials composed of only S-shaped resonators," Phys. Rev. E, vol. 70, p. 57605, 2004.

[7] D. R. A. K. Smith, "Negative refractive index in left-handed materials," Phys. Rev. Lett., vol. 85, pp. 2933-2936, 2000.

[8] W. Cai, U. K. Chettiar, A. V. Kildishev, et al., "Optical cloaking with metamaterials," Nat Photon., vol. 1, pp. 224-227, 2007.

[9] D. Schurig, J. J. Mock, B. J. Justice, et al., "Metamaterial electromagnetic cloak at microwave frequencies," Science, vol. 314, pp. 977-980, 2006.

[10] H. Chen, W. J. Padilla, J. M. O. Zide, et al., "Active terahertz metamaterial devices," Nature, vol. 444, pp. 597-600, 2006.

[11] H. Tao, W. J. Padilla, X. Zhang, and R. D. Averitt, "Recent progress in electromagnetic metamaterial devices for terahertz applications," IEEE J. Sel. Top. Quant., vol. 17, pp. 92-101, 2011.

[12] Q. Wang, E. T. F. Rogers, B. Gholipour, et al., "Optically reconfigurable metasurfaces and photonic devices based on phase change materials," Nat Photonics, vol. 10, pp. 60-65, 2016.

[13] N. Yu and F. Capasso, "Flat optics with designer metasurfaces," Nat. Mater., vol. 13, pp. 139-150, 2014.

[14] N. Yu, P. Genevet, F. Aieta, et al., "Flat optics: controlling wavefronts with optical antenna metasurfaces," IEEE J. Sel. Top. Quant., vol. 19, p. 4700423, 2013.

[15] A. Alù and N. Mohammadi Estakhri, "Wave-front transformation with gradient metasurfaces," Phys. Rev. X, vol. 6, p. 41008, 2016.

[16] S. M. Kamali, E. Arbabi, A. Arbabi, et al., "A review of dielectric optical metasurfaces for wavefront control," Nanophotonics, vol. 7, pp. 1041-1068, 2018.

[17] N. Yu, P. Genevet, M. A. Kats, et al., "Light propagation with phase discontinuities: generalized laws of reflection and refraction," Science, vol. 334, pp. 333-337, 2011.

[18] Z. Liu, Z. Li, Z. Liu, et al., "High-performance broadband circularly polarized beam deflector by mirror effect of multinanorod metasurfaces," Adv. Funct. Mater., vol. 25, pp. 5428-5434, 2015.

[19] Q. Zhang, M. Li, T. Liao, et al., "Design of beam deflector, splitters, wave plates and metalens using photonic elements with dielectric metasurface," Opt. Commun., vol. 411, pp. 93$100,2018$.

[20] X. Su, C. Ouyang, N. Xu, et al., "Active metasurface terahertz deflector with phase discontinuities," Opt. Express, vol. 23, pp. 27152-27158, 2015.

[21] X. Ni, S. Ishii, A. V. Kildishev, et al., "Ultra-thin, planar, Babinetinverted plasmonic metalenses," Light Sci. Appl., vol. 2, p. e72, 2013.

[22] F. Lemoult, M. Fink, and G. Lerosey, "Far-field sub-wavelength imaging and focusing using a wire medium based resonant metalens," Wave Random Complex, vol. 21, pp. 614-627, 2011.

[23] Y. Huang, W. T. Chen, W. Tsai, et al., "Aluminum plasmonic multicolor meta-hologram,” Nano Lett., vol. 15, pp. 3122-3127, 2015.
[24] X. Luo, Y. Hu, X. Li, et al., “Integrated Metasurfaces with Microprints and Helicity-Multiplexed Holograms for Real-Time Optical Encryption," Adv. Opt. Mater., vol. 8, p. 1902020, 2020.

[25] W. Wan, W. Qiao, D. Pu, et al., "Holographic sampling display based on metagratings," iScience, vol. 23, p. 100773, 2020.

[26] F. Yue, D. Wen, J. Xin, et al., "Vector vortex beam generation with a single plasmonic metasurface," ACS Photonics, vol. 3, pp. 1558-1563, 2016.

[27] E. Karimi, S. A. Schulz, I. De Leon, et al., "Generating optical orbital angular momentum at visible wavelengths using a plasmonic metasurface," Light Sci. Appl., vol. 3, p. e167, 2014.

[28] H. Yang, Z. Chen, Q. Liu, et al., "Near-field orbital angular momentum generation and detection based on spin-orbit interaction in gold metasurfaces," Adv. Theory Simul., vol. 2, p. 1900133, 2019.

[29] A.Krasnok, S.Makarov, M.Petrov, R.Savelev, P.Belov, Y.Kivshar, Towards All-Dielectric Metamaterials and Nanophotonics, Proc. SPIE 9502, Metamaterials X, 950203 (5 May 2015); Proceedings volume 9502, Metamaterials X; 950203 (2015) Event: Prague, Czech Republic, SPIE Optics + Optoelectronics, 2015.

[30] Y. F. Yu, A. Y. Zhu, R. Paniagua-Domínguez, et al., "Hightransmission dielectric metasurface with $2 \pi$ phase control at visible wavelengths," Laser Photonics Rev., vol. 9, pp. 412-418, 2015.

[31] A. Zhan, S. Colburn, R. Trivedi, et al., "Low-contrast dielectric metasurface optics,” ACS Photonics, vol. 3, pp. 209-214, 2016.

[32] M. I. Shalaev, J. Sun, A. Tsukernik, et al., "High-efficiency alldielectric metasurfaces for ultracompact beam manipulation in transmission mode," Nano Lett., vol. 15, pp. 6261-6266, 2015.

[33] B. H. Chen, P. C. Wu, V. Su, et al., "GaN metalens for pixel-level full-color routing at visible light," Nano Lett., vol. 17, pp. 63456352, 2017.

[34] S. Colburn, A. Zhan, E. Bayati, et al., "Broadband transparent and CMOS-compatible flat optics with silicon nitride metasurfaces [Invited]," Opt. Mater. Express., vol. 8, pp. 23302344, 2018.

[35] A. Arbabi, E. Arbabi, S. M. Kamali, et al., "Miniature optical planar camera based on a wide-angle metasurface doublet corrected for monochromatic aberrations," Nat. Commun., vol. 7, p. 13682, 2016.

[36] E. Arbabi, A. Arbabi, S. M. Kamali, et al., "MEMS-tunable dielectric metasurface lens," Nat. Commun., vol. 9, p. 812, 2018.

[37] L. Liu, X. Zhang, M. Kenney, et al., "Broadband metasurfaces with simultaneous control of phase and amplitude," Adv. Mater., vol. 26, pp. 5031-5036, 2014.

[38] G. Lee, G. Yoon, S. Lee, et al., "Complete amplitude and phase control of light using broadband holographic metasurfaces," Nanoscale, vol. 10, pp. 4237-4245, 2018.

[39] Z. Yang, Y. Chen, Y. Zhou, et al., "Microscopic interference fullcolor printing using grayscale-patterned fabry-perot resonance cavities," Adv. Opt. Mater., vol. 5, p. 1700029, 2017.

[40] P. Dai, Y. Wang, X. Zhu, et al., "Transmissive structural color filters using vertically coupled aluminum nanohole/nanodisk array with a triangular-lattice," Nanotechnology, vol. 29, p. 395202, 2018.

[41] K. Kumar, H. Duan, R. S. Hegde, et al., "Printing colour at the optical diffraction limit," Nat. Nanotechnol., vol. 7, pp. 557-561, 2012.

[42] Y. Wang, M. Zheng, Q. Ruan, et al., "Stepwise-nanocavityassisted transmissive color filter array microprints," Research (Wash D C), vol. 2018, 2018, Art no. 8109054. 
[43] B. Sain, C. Meier, and T. Zentgraf, "Nonlinear optics in alldielectric nanoantennas and metasurfaces: a review," Adv. Photonics, vol. 1, p. 24002, 2019.

[44] J. Deng, Y. Yang, J. Tao, et al., "Spatial frequency multiplexed meta-holography and meta-nanoprinting," ACS Nano, vol. 13, pp. 9237-9246, 2019.

[45] W. Zang, Q. Yuan, R. Chen, et al., "Chromatic dispersion manipulation based on metalenses," Adv. Mater., vol. 1904935, 2019, https://doi.org/10.1002/adma.201904935.

[46] Y. Intaravanne and X. Chen, "Recent advances in optical metasurfaces for polarization detection and engineered polarization profiles," Nanophotonics, 2020. (published online ahead of print), 20190479.

[47] L. Huang, D. R. Chowdhury, S. Ramani, et al., "Impact of resonator geometry and its coupling with ground plane on ultrathin metamaterial perfect absorbers," Appl. Phys. Lett., vol. 101, p. 101102, 2012.

[48] X. Liu, C. Lan, B. Li, et al., "Dual band metamaterial perfect absorber based on artificial dielectric "molecules", Sci. Rep. UK, vol. 6, p. 28906, 2016.

[49] X. Zhu, W. Yan, U. Levy, et al., "Resonant laser printing of structural colors on high-index dielectric metasurfaces," Sci. Adv., vol. 3, p. e1602487, 2017.

[50] S. Sun, Z. Zhou, C. Zhang, et al., "All-dielectric full-color printing with TiO2 metasurfaces," ACS Nano, vol. 11, pp. 4445-4452, 2017.

[51] H. Butt, Y. Montelongo, T. Butler, et al., "Carbon nanotube based high resolution holograms," Adv. Mater., vol. 24, pp. 331-336, 2012.

[52] S. Liu, M. B. Sinclair, S. Saravi, et al., "Resonantly enhanced secondharmonic generation using III-V semiconductor all-dielectric metasurfaces," Nano Lett., vol. 16, pp. 5426-5432, 2016.

[53] S. Liu, P. P. Vabishchevich, A. Vaskin, et al., "An all-dielectric metasurface as a broadband optical frequency mixer," Nat. Commun., vol. 9, p. 2507, 2018.

[54] S. Keren-Zur, O. Avayu, L. Michaeli, et al., "Nonlinear beam shaping with plasmonic metasurfaces," ACS Photonics, vol. 3, pp. 117-123, 2016.

[55] C. Schlickriede, N. Waterman, B. Reineke, et al., "Imaging through nonlinear metalens using second harmonic generation," Adv. Mater., vol. 30, p. 1703843, 2018.

[56] Y. Gao, Y. Fan, Y. Wang, et al., "Nonlinear holographic all-dielectric metasurfaces," Nano Lett., vol. 18, pp. 8054-8061, 2018.

[57] O. Avayu, E. Almeida, Y. Prior, et al., "Composite functional metasurfaces for multispectral achromatic optics," Nat. Commun., vol. 8, pp. 1-7, 2017.

[58] W. T. Chen, A. Y. Zhu, V. Sanjeev, et al., "A broadband achromatic metalens for focusing and imaging in the visible," Nat. Nanotechnol., vol. 13, pp. 220-226, 2018.

[59] S. Wang, P. C. Wu, V. Su, et al., "A broadband achromatic metalens in the visible," Nat. Nanotechnol., vol. 13, pp. 227-232, 2018.

[60] Z. Zhao, M. Pu, H. Gao, et al., "Multispectral optical metasurfaces enabled by achromatic phase transition," Sci. Rep. UK, vol. 5, p. 15781, 2015.

[61] W. Zhao, B. Liu, H. Jiang, et al., "Full-color hologram using spatial multiplexing of dielectric metasurface," Opt. Lett., vol. 41, p. 147, 2016.

[62] J. Zheng, Z. Ye, N. Sun, et al., "Highly anisotropic metasurface: a polarized beam splitter and hologram," Sci. Rep. UK, vol. 4, p. 6491, 2014.

[63] S. Jahani and Z. Jacob, "All-dielectric metamaterials," Nat. Nanotechnol., vol. 11, pp. 23-36, 2016.
[64] S. Kruk, B. Hopkins, I. I. Kravchenko, et al., "Invited Article: broadband highly efficient dielectric metadevices for polarization control," APL Photonics, vol. 1, p. 30801, 2016.

[65] X. Song, L. Huang, C. Tang, et al., "Selective diffraction with complex amplitude modulation by dielectric metasurfaces," Adv. Opt. Mater., vol. 6, p. 1701181, 2018.

[66] R. C. Jones, "A new calculus for the treatment of optical Systemsl. Description and discussion of the calculus,"J. Opt. Soc. Am., vol. 31, pp. 488-493, 1941.

[67] A. Arbabi, Y. Horie, M. Bagheri, et al., "Dielectric metasurfaces for complete control of phase and polarization with subwavelength spatial resolution and high transmission," Nat. Nanotechnol., vol. 10, pp. 937-943, 2015.

[68] Y. Yang, W. Wang, P. Moitra, et al., "Dielectric meta-reflectarray for broadband linear polarization conversion and optical vortex generation," Nano Lett., vol. 14, pp. 1394-1399, 2014.

[69] D. Lin, P. Fan, E. Hasman, et al., "Dielectric gradient metasurface optical elements," Science, vol. 345, pp. 298-302, 2014.

[70] B. Shen, P. Wang, R. Polson, et al., "Ultra-high-efficiency metamaterial polarizer," Optica, vol. 1, pp. 356-360, 2014.

[71] A. Arbabi, Y. Horie, M. Bagheri, et al., "Dielectric metasurfaces for complete control of phase and polarization with subwavelength spatial resolution and high transmission," Nat. Nanotechnol., vol. 10, pp. 937-943, 2015.

[72] S. Kruk, B. Hopkins, I. I. Kravchenko, et al., "Invited Article: broadband highly efficient dielectric metadevices for polarization control," APL Photonics, vol. 1, p. 30801, 2016.

[73] J. Hu, X. Zhao, Y. Lin, et al., "All-dielectric metasurface circular dichroism waveplate," Sci. Rep. UK, vol. 7, p. 41893, 2017.

[74] Z. Ma, Y. Li, Y. Li, et al., "All-dielectric planar chiral metasurface with gradient geometric phase," Opt. Express, vol. 26, pp.60676078, 2018

[75] Q. Zhang, M. Li, T. Liao, et al., "Design of beam deflector, splitters, wave plates and metalens using photonic elements with dielectric metasurface," Opt. Commun., vol. 411, pp. 93100, 2018.

[76] S. Gao, C. Park, S. Lee, et al., "All-dielectric metasurfaces for simultaneously realizing polarization rotation and wavefront shaping of visible light," Nanoscale, vol. 11, pp. 4083-4090, 2019.

[77] X. Cai, Z. Li, Y. Kong, et al., "Tunable polarization rotation using non-chiral all-dielectric metasurfaces," Optik, p. 163769, 2019.

[78] D. Lin, P. Fan, E. Hasman, et al., "Dielectric gradient metasurface optical elements," Science, vol. 345, pp. 298-302, 2014.

[79] Q. Zhang, M. Li, T. Liao, et al., "Design of beam deflector, splitters, wave plates and metalens using photonic elements with dielectric metasurface," Opt. Commun., vol. 411, pp. 93100, 2018.

[80] Y. Wang, W. Zhu, C. Zhang, et al., "Ultra-compact visible light depolarizer based on dielectric metasurface," Appl. Phys. Lett., vol. 116, p. 51103, 2020.

[81] M. Khorasaninejad and K. B. Crozier, "Silicon nanofin grating as a miniature chirality-distinguishing beam-splitter," Nat. Commun., vol. 5, p. 5386, 2014.

[82] X. Chen, L. Huang, H. Mühlenbernd, et al., "Dual-polarity plasmonic metalens for visible light," Nat. Commun., vol. 3, p. 1198, 2012.

[83] J. Zhou, H. Qian, C. Chen, et al., "Optical edge detection based on high-efficiency dielectric metasurface," Proc. Natl. Acad. Sci., vol. 116, pp. 11137-11140, 2019. 
[84] Z. Li, Q. Dai, M. Q. Mehmood, et al., "Full-space cloud of random points with a scrambling metasurface," Light Sci. Appl., vol. 7, pp. 1-8, 2018.

[85] M. Khorasaninejad, W. Zhu, and K. B. Crozier, "Efficient polarization beam splitter pixels based on a dielectric metasurface," Optica, vol. 2, pp. 376-382, 2015.

[86] Zhongyi Guo LZFS, "Dielectric metasurface based highefficiency polarization splitters," RSC Adv., vol. 7, pp. 98759879, 2017.

[87] Z. Guo, L. Zhu, K. Guo, et al., "High-order dielectric metasurfaces for high-efficiency polarization beam splitters and optical vortex generators," Nanoscale Res. Lett., vol. 12, pp. 1-8, 2017.

[88] J. Li, C. Liu, T. Wu, et al., "Efficient polarization beam splitter based on all-dielectric metasurface in visible region," Nanoscale Res. Lett., vol. 14, p. 34, 2019.

[89] H. Kwon, E. Arbabi, S. M. Kamali, et al., "Single-shot quantitative phase gradient microscopy using a system of multifunctional metasurfaces," Nat. Photonics, vol. 14, pp. 109-114, 2020.

[90] G. Zheng, G. Liu, M. G. Kenney, et al., "Ultracompact highefficiency polarising beam splitter based on silicon nanobrick arrays," Opt. Express, vol. 24, pp. 6749-6757, 2016.

[91] M. Khorasaninejad and F. Capasso, "Metalenses: versatile multifunctional photonic components," Science, vol. 358, p. 6367, 2017.

[92] P. Lalanne and P. Chavel, "Metalenses at visible wavelengths: past, present, perspectives," Laser Photonics Rev., vol. 11, p. 1600295, 2017.

[93] E. Schonbrun, K. Seo, and K. B. Crozier, "Reconfigurable imaging systems using elliptical nanowires," Nano Lett., vol. 11, pp. 4299-4303, 2011.

[94] R. Lin and X. Li, “Multifocal metalens based on multilayer Pancharatnam-Berry phase elements architecture," Opt. Lett., vol. 44, p. 2819, 2019.

[95] S. Tian, H. Guo, J. Hu, et al., "Dielectric longitudinal bifocal metalens with adjustable intensity and high focusing efficiency," Opt. Express, vol. 27, p. 680, 2019.

[96] S. Gao, C. S. Park, C. Zhou, et al., "Twofold polarizationselective all-dielectric trifoci metalens for linearly polarized visible light," Adv. Opt. Mater., vol. 7, p. 1900883, 2019.

[97] X. Zang, H. Ding, Y. Intaravanne, et al., "A multi-foci metalens with polarization-rotated focal points," Laser Photonics Rev., vol. 13, p. 1900182, 2019.

[98] M. D. Aiello, A. S. Backer, A. J. Sapon, et al., "Achromatic varifocal metalens for the visible spectrum," ACS Photonics, vol. 6, pp. 2432-2440, 2019.

[99] S. Li, X. Li, G. Wang, et al., "Multidimensional manipulation of photonic spin Hall effect with a single-layer dielectric metasurface," Adv. Opt. Mater., vol. 7, p. 1801365, 2018.

[100] H. Liang, Q. Lin, X. Xie, et al., "Ultrahigh numerical aperture metalens at visible wavelengths," Nano Lett., vol. 18, pp. 44604466, 2018.

[101] M. Khorasaninejad, W. T. Chen, R. C. Devlin, et al., "Metalenses at visible wavelengths: diffraction-limited focusing and subwavelength resolution imaging," Science, vol. 352, pp. 1190-1194, 2016.

[102] F. Zhang, H. Yu, J. Fang, et al., “Efficient generation and tight focusing of radially polarized beam from linearly polarized beam with all-dielectric metasurface," Opt. Express, vol. 24, p. $6656,2016$.
[103] O. Mandula, M. Kielhorn, K. Wicker, et al., “Line scan-structured illumination microscopy super-resolution imaging in thick fluorescent samples," Opt. Express, vol. 20, p. 24167, 2012.

[104] R. Zuo, W. Liu, H. Cheng, et al., "Breaking the diffraction limit with radially polarized light based on dielectric metalenses," Adv. Opt. Mater., vol. 6, p. 1800795, 2018.

[105] D. Gabor, “A new microscopic principle," Nature, vol. 161, pp. 777-778, 1948.

[106] K. Huang, H. Liu, Garcia-Vidal FJ, et al., “Ultrahigh-capacity nonperiodic photon sieves operating in visible light," Nat. Commun., vol. 6, pp. 1-7, 2015.

[107] B. Desiatov, N. Mazurski, Y. Fainman, et al., "Polarization selective beam shaping using nanoscale dielectric metasurfaces," Opt. Express, vol. 23, p. 22611, 2015.

[108] M. Khorasaninejad, A. Ambrosio, P. Kanhaiya, et al., "Broadband and chiral binary dielectric meta-holograms," Sci. Adv., vol. 2, p. e1501258, 2016.

[109] Balthasar Mueller JP, N. A. Rubin, R. C. Devlin, et al., "Metasurface polarization optics: independent phase control of arbitrary orthogonal states of polarization," Phys. Rev. Lett., vol. 118, p. 113901, 2017.

[110] R. Zhao, B. Sain, Q. Wei, et al., "Multichannel vectorial holographic display and encryption. Light," Sci. Appl., vol. 7, p. 95, 2018.

[111] B. Wang, F. Dong, Q. Li, et al., "Visible-frequency dielectric metasurfaces for multiwavelength Achromatic and highly dispersive holograms," Nano Lett., vol. 16, pp. 5235-5240, 2016.

[112] L. Jin, Z. Dong, S. Mei, et al., "Noninterleaved metasurface for (26-1) spin- and wavelength-encoded holograms," Nano Lett., vol. 18, pp. 8016-8024, 2018.

[113] Y. Hu, L. Li, Y. Wang, et al., "Trichromatic and tripolarizationchannel holography with non-interleaved dielectric metasurface," Nano Lett., vol. 20, pp. 994-1002, 2019.

[114] E. Arbabi, S. M. Kamali, A. Arbabi, et al., "Vectorial holograms with a dielectric metasurface: ultimate polarization pattern generation," ACS Photonics, vol. 6, pp. 2712-2718, 2019.

[115] K. Kumar, H. Duan, R. S. Hegde, et al., "Printing colour at the optical diffraction limit," Nat. Nanotechnol., vol. 7, pp. 557-561, 2012.

[116] S. Sun, Z. Zhou, C. Zhang, et al., "All-dielectric full-color printing with TiO2 metasurfaces," ACS Nano, vol. 11, pp. 44454452, 2017.

[117] Q. Dai, L. Deng, J. Deng, et al., “Ultracompact, high-resolution and continuous grayscale image display based on resonant dielectric metasurfaces," Opt. Express, vol. 27, p. 27927, 2019.

[118] R. Zhao, L. Huang, C. Tang, et al., "Nanoscale polarization manipulation and encryption based on dielectric metasurfaces," Adv. Opt. Mater., vol. 6, p. 1800490, 2018.

[119] X. Zang, F. Dong, F. Yue, et al., "Polarization encoded color image embedded in a dielectric metasurface," Adv. Mater., vol. 30, p. 1707499, 2018.

[120] J. Deng, L. Deng, Z. Guan, et al., "Multiplexed anticounterfeiting meta-image displays with single-sized nanostructures," Nano Lett., vol. 20, pp. 1830-1838, 2020.

[121] B. Yang, W. Liu, Z. Li, et al., "Polarization-sensitive structural colors with hue-and-saturation tuning based on all-dielectric nanopixels," Adv. Opt. Mater., vol. 6, p. 1701009, 2018.

[122] S. Gao, C. Park, S. Lee, et al., "A highly efficient bifunctional dielectric metasurface enabling polarization-tuned focusing and deflection for visible light," Adv. Opt. Mater., vol. 7, p. 1801337, 2019. 
[123] Y. Hu, X. Luo, Y. Chen, et al., "3D-Integrated metasurfaces for full-colour holography," Light Sci. Appl., vol. 8, pp. 1-9, 2019.

[124] G. Yoon, D. Lee, K. T. Nam, et al., “““Crypto-Display” in dualmode metasurfaces by simultaneous control of phase and spectral responses," ACS Nano, vol. 12, pp. 6421-6428, 2018.

[125] Q. Wei, B. Sain, Y. Wang, et al., "Simultaneous spectral and spatial modulation for color printing and holography using all-dielectric metasurfaces," Nano Lett., vol. 19, pp. 8964-8971, 2019.

[126] A. C. Overvig, S. Shrestha, S. C. Malek, et al., "Dielectric metasurfaces for complete and independent control of the optical amplitude and phase," Light Sci. Appl., vol. 8, p. 92, 2019.

[127] Y. Bao, Y. Yu, H. Xu, et al., "Full-colour nanoprint-hologram synchronous metasurface with arbitrary hue-saturationbrightness control," Light Sci. Appl., vol. 8, p. 95, 2019.

[128] S. Wei, Z. Yang, and M. Zhao, "Design of ultracompact polarimeters based on dielectric metasurfaces," Opt. Lett., vol. 42, p. 1580, 2017.

[129] K. Guo, H. Xu, Z. Peng, et al., "High-efficiency full-vector polarization analyzer based on GaN metasurface," IEEE Sens. J., vol. 19, pp. 3654-3659, 2019.

[130] E. Arbabi, S. M. Kamali, A. Arbabi, et al., "Full-Stokes imaging polarimetry using dielectric metasurfaces," ACS Photonics, vol. 5, pp. 3132-3140, 2018.

[131] Z. Yang, Z. Wang, Y. Wang, et al., "Generalized Hartmann-Shack array of dielectric metalens sub-arrays for polarimetric beam profiling," ACS Photonics, vol. 9, pp. 1-7, 2018.

[132] X. Zhang, S. Yang, W. Yue, et al., "Direct polarization measurement using a multiplexed Pancharatnam-Berry metahologram," Optica, vol. 6, p. 1190, 2019.

[133] M. Khorasaninejad, W. T. Chen, A. Y. Zhu, et al., "Multispectral chiral imaging with a metalens," Nano Lett., vol. 16, pp. 4595-4600, 2016.

[134] C. Yan, X. Li, M. Pu, et al., "Midinfrared real-time polarization imaging with all-dielectric metasurfaces," Appl. Phys. Lett., vol. 114, p. 161904, 2019.

[135] N. A. Rubin, G. D’Aversa, P. Chevalier, et al., "Matrix Fourier optics enables a compact full-Stokes polarization camera," Science (New York, N.Y.), vol. 365, p. 43, 2019.

[136] C. Rosales-Guzmán, B. Ndagano, and A. Forbes, "A review of complex vector light fields and their applications," J. Opt. UK, vol. 20, p. 123001, 2018.

[137] H. Chen, Z. Chen, Q. Li, et al., Generation of vector beams based on dielectric metasurfaces, 2015.

[138] Y. He, Y. Li, J. Liu, et al., "Switchable phase and polarization singular beams generation using dielectric metasurfaces," Sci. Rep. UK, vol. 7, pp. 6810-6814, 2017.

[139] E. Maguid, I. Yulevich, M. Yannai, et al., "Multifunctional interleaved geometric-phase dielectric metasurfaces," Light Sci. Appl., vol. 6, p. e17027, 2017.

[140] R. Wang, S. He, S. Chen, et al., "Electrically driven generation of arbitrary vector vortex beams on the hybrid-order Poincaré sphere," Opt. Lett., vol. 43, p. 3570, 2018.
[141] Y. Liu, Y. Ke, J. Zhou, et al., “Generation of perfect vortex and vector beams based on Pancharatnam-Berry phase elements," Sci. Rep. UK, vol. 7, 2017, https://doi.org/10. 1038/srep44096.

[142] Y. Bao, J. Ni, and C. W. Qiu, “A minimalist single-layer metasurface for arbitrary and full control of vector vortex beams," Adv. Mater., p. 1905659, 2019, https://doi.org/10. 1002/adma.201905659.

[143] G. Molina-Terriza, J. P. Torres, and L. Torner, "Twisted photons," Nat. Phys., vol. 3, pp. 305-310, 2007.

[144] M. Chen, W. Gao, H. Liu, et al., "Polarization controllable generation of flat superimposed OAM states based on metasurface," Opt. Express, vol. 27, p. 20133, 2019.

[145] R. C. Devlin, A. Ambrosio, N. A. Rubin, et al., "Arbitrary spin-toorbital angular momentum conversion of light," Science (New York, N.Y.), vol. 358, pp. 896-901, 2017.

[146] R. C. Devlin, A. Ambrosio, D. Wintz, et al., "Spin-to-orbital angular momentum conversion in dielectric metasurfaces," Opt. Express, vol. 25, p. 377, 2017.

[147] G. Biener, A. Niv, V. Kleiner, et al., "Formation of helical beams by use of Pancharatnam-Berry phase optical elements," Opt. Lett., vol. 27, p. 1875, 2002.

[148] T. Stav, A. Faerman, E. Maguid, et al., "Quantum entanglement of the spin and orbital angular momentum of photons using metamaterials," Science, vol. 361, pp. 11011104, 2018.

[149] K. Wang, J. G. Titchener, S. S. Kruk, et al., "Quantum metasurface for multiphoton interference and state reconstruction," Science, vol. 361, pp. 1104-1108, 2018.

[150] P. Georgi, M. Massaro, K. H. Luo, et al., "Metasurface interferometry toward quantum sensors," Light Sci. Appl., vol. 8, p. 70, 2019.

[151] J. Jiang and J. A. Fan, "Global optimization of dielectric metasurfaces using a physics-driven neural network," Nano Lett., vol. 19, pp. 5366-5372, 2019.

[152] J. Jiang and J. A. Fan, "Simulator-based training of generative neural networks for the inverse design of metasurfaces," Nanophotonics, 2019. (published online ahead of print), 20190330.

[153] D. Liu, Y. Tan, E. Khoram, et al., "Training deep neural networks for the inverse design of nanophotonic structures," ACS Photonics, vol. 5, pp. 1365-1369, 2018.

[154] Y. Dong, Z. Xu, N. Li, et al., "Si metasurface half-wave plates demonstrated on a 12-inch CMOS platform," Nanophotonics, vol. 9, pp. 149-157, 2020.

[155] T. Phan, D. Sell, E. W. Wang, et al., "High-efficiency, large-area, topology-optimized metasurfaces," Light Sci. Appl., vol. 8, p. 48, 2019.

[156] A. She, S. Zhang, S. Shian, et al., "Large area metalenses: design, characterization, and mass manufacturing," Opt. Express, vol. 26, pp. 1573-1585, 2018. 